\title{
A NEW FOUNDATION OF THE PRO JECTIVE DIFFERENTIAL THEORY OF CURVES IN FIVE-DIMENSIONAL SPACE
}

\author{
BY \\ SU-CHENG CHANG(1)
}

Introduction. The purpose of the present paper is to develop a purely geometric theory of the projective differential geometry of curves in a space of five dimensions, the methods hitherto adopted by various authors $\left({ }^{2}\right)$ being more or less analytic and artificial.

The projective differential theory of plane curves is one of the cornerstones upon which our theory of curves in five dimensions rests. The problem of finding a covariant figure to associate projectively with the neighborhood of order five at an ordinary point of a plane curve is an interesting one as has been pointed out by Levi-Civita and Fubini [9]. We have succeeded in solving this problem in an elementary way. Then using neighborhoods of order six we have obtained, besides a covariant triangle, three covariant points $I_{i}$ $(i=1,2,3)$ any one of which can be selected as unit point.

In five-dimensional space the osculating plane $p$ at an ordinary point $P$ of a curve $\Gamma$ intersects the developable hypersurface of $\Gamma$ in a plane curve $C$, of which $P$ is either an ordinary point or a $k$-ic $(k=6,7,8)$ point [2]. In any case a covariant unit point $I$ and a covariant triangle $\left\{P P_{1} P_{2}\right\}$ can be determined in $p, P P_{1}$ being the tangent to $\Gamma$ at $P$.

When a plane $p$, passing through $P P_{1}$ and lying in the osculating threespace of $\Gamma$ at $P$, rotates about $P P_{1}$, the Bompiani osculant $O_{6}[1]$, associated with the point of inflexion $P$ of the projection produced by projecting $\Gamma$ on the plane $p$ from the point $\alpha P+\beta P_{2}$, constitutes a generator of a covariant quadric $Q_{3}$. Since $P, P_{1}, P_{2}$ are three vertices of a quadrilateral on $Q_{3}$, we may take the fourth vertex for $P_{3}$. In a similar way we define two other vertices $P_{4}$ and $P_{5}$ of a covariant pyramid $\left\{P P_{1} P_{2} P_{3} P_{4} P_{5}\right\}$ and two other covariant quadrics $Q_{4}$ and $Q_{5}$ which pass through the quadrilaterals $P P_{1} P_{3} P_{4} P$ and $P P_{1} P_{4} P_{5} P$ respectively.

Let $I$ be the unit point in the plane $P P_{1} P_{2}$; then the unit point in the space may be defined by the fact that the quadrics $Q_{k}(k=3,4,5)$ pass through $P+P_{1}+P_{2}+P_{k}$ respectively.

This accomplished, the projective Frenet-Serret formulae follow immediately from the canonical expansions of $\Gamma$. Our method has the advantage

Presented to the Society, November 24, 1945; received by the editors November 6, 1944.

(1) The stay of the author in the National University of Chekiang was made possible by the Board of Trustees of the indemnity funds remitted by the British government.

(2) Wilczynski [15], Sannia [10], Su [11, 12]. Numbers in brackets refer to the Bibliography at the end of the paper. 
that the geometric significance of the projective invariants of the curve can easily be brought to light.

\section{Chapter I. The projective differential theory of Plane curves}

In this chapter we shall discuss some results useful for the study of curves in a five-dimensional projective space. In $\$ 1$ a covariant triangle of reference and a covariant unit point are constructed by means of the neighborhood of order six at a point of an ordinary plane curve. This leads us in $\$ 2$ to reconstruct Lane's [8] canonical expansion of a plane curve at its sextactic point.

1. A new projective differential theory of plane curves. In this section we study plane curves in the neighborhood of an ordinary point, obtaining a canonical expansion, the Frenet-Serret formulae and an interpretation of the invariants.

1.1. The covariant triangle of reference. Let $x$ and $y$ be nonhomogeneous coordinates of a point in a plane. If $x=x(u), y=y(u)$ are the parametric equations of a plane curve $C$, the expansion of $C$ at an ordinary point $P$ of $C$ with respect to a local triangle of reference $\left\{P P_{1} P_{2}\right\}$ is

$$
y=a x^{2}+b x^{3}+c x^{4}+d x^{5}+e x^{6}+f x^{7}+(8),
$$

where $P P_{1}$ is the tangent to $C$ at $P$. We take $P_{2}$ on the osculating conic $C_{2}$ of $C$ at $P$ and $P_{1} P_{2}$ as the tangent to the conic $C_{2}$ at $P_{2}$, so that (1) reduces to

$$
y=a x^{2}+d x^{5}+e x^{6}+f x^{7}+(8) .
$$

It is well known that there exists a bundle of seven-point cubics of $C$ at $P$ : (3) $\alpha y\left(y-a x^{2}\right)+\beta\left(y-a x^{2}-d x y^{2} / a^{2}-e y^{3} / a^{3}\right)+\gamma\left(a x^{3}-x y+d y^{3} / a^{3}\right)=0$, where $\alpha, \beta, \gamma$ are arbitrary constants. $y=0$ and $y-a x^{2}=0$ respectively intersect (3), besides $P$, at a pair of related points

$$
(\beta / \gamma, 0)
$$

and

$$
\left(\beta d /(\gamma d-\beta c), a \beta^{2} d^{2} /(\gamma d-\beta e)^{2}\right),
$$

whose join passes through a fixed point

$$
\left(d / e, a d^{2} / e^{2}\right) \text {. }
$$

For $d \neq 0$, we select (6) for $P_{2}$ and reduce (2) to

$$
y=a x^{2}+d x^{5}+f x^{7}+(8) .
$$

1.2. Covariant figure associated with an ordinary point of a plane curve. Let $x^{1}, x^{2}, x^{3}$ be the homogeneous coordinates of a point defined by

$$
x=x^{1} / x^{3}, \quad y=x^{2} / x^{3} .
$$


The polar line of $A(\alpha, 0,1)$ with respect to $C_{2}$,

$$
x^{2}=2 a \alpha x^{1}
$$

intersects $C_{2}$ at $(0,0,1)$ and

$$
A^{\prime}(1,2 a \alpha, 1 / 2 \alpha)
$$

respectively. At $P$ a six-point cubic of $C$ can be determined to be tangent to the lines (7) and $A A^{\prime}$,

$$
-2 a \alpha x^{1}+x^{2} / 2+2 a \alpha^{2} x^{8}=0,
$$

at $(8)$ and $(\alpha, 0,1)$ respectively; its equation is found to be

$$
\begin{aligned}
(y-2 a \alpha x)^{2}\left(-2 a \alpha x+y / 2+2 a \alpha^{2}\right) & +(y-2 a \alpha x)\left(-4 d \alpha^{3} y^{2} / a\right) \\
& -2 y\left(-2 a \alpha x+y / 2+2 a \alpha^{2}\right)^{2}=0 .
\end{aligned}
$$

As $\alpha$ varies the envelope of the cubic (10) is a covariant figure:

$$
4 a^{5}\left(a x^{2}-y\right)^{3}+d^{2} y^{6}=0 .
$$

1.3. The canonical expansion. The tangent of the conic $y-a x^{2}=0$ at the covariant point (6) intersects $y=0$ at $(d / 2 e, 0,1)$. A triangle of vertices $P$, $(6)$, and $(d / 2 e, 0,1)$ is called a fundamental triangle. We denote (6) and $(d / 2 e, 0,1)$ by $P_{2}$ and $P_{1}$ respectively. If the fundamental triangle is taken for reference, the expansion of the curve $C$ at $P$ then becomes

$$
y=a x^{2}+d x^{5}+f x^{7}+(8) \text {. }
$$

As we have given in $\$ 1.2$, the two consecutive points $(1,0,0)$ and $(\alpha, 0,1)$ $(\alpha \rightarrow \infty)$ on $P P_{1}$ correspond to the cubics

$$
-d x y^{2}-a^{3} x^{2}+a^{2} y=0
$$

and (10) respectively. They intersect at the points on the cubic

$$
a^{3} x^{3}-a^{2} x y+d y^{3} / 2 a=0,
$$

namely, $P$ and the points of coordinates

$$
\left(\omega^{k}\left(d / 2 a^{4}\right)^{1 / 3}, \quad 0, \quad 2 a \omega^{2 k}\left(d / 2 a^{4}\right)^{2 / 8}\right) \quad\left(k=1,2,3, \quad \omega^{2}=1, \omega \neq 1\right) .
$$

If one of the latter is taken for unit point $I$, then we have

$$
d=1 / 8, \quad a=1 / 2 .
$$

The coordinate system thus obtained will be called the canonical coordinate system, and the coordinates of a point referred to it the canonical coordinates.

The canonical expansion of $C$ at $P$ now becomes

$$
y=x^{2} / 2+x^{5} / 8+k(u) x^{7}+(8) \text {. }
$$

1.4. Projective Frenet-Serret formulae. Let $\left(x^{1}(u), x^{2}(u), x^{3}(u)\right),\left(x_{1}^{1}(u)\right.$, 
$\left.x_{1}^{2}(u), x_{1}^{3}(u)\right)$, and $\left(x_{2}^{1}(u), x_{2}^{2}(u), x_{2}^{3}(u)\right)$ be the coordinates of the vertices $P, P_{1}, P_{2}$ of the fundamental triangle. In order to preserve $x^{j}(u)+x_{1}^{j}(u)+x_{2}^{j}(u)$ $(j=1,2,3)$ as the selected unit point associated with the canonical triangle of reference $\left\{P(u), P_{1}(u), P_{2}(u)\right\}$, we are unable to multiply the coordinates of the three points $P(u), P_{1}(u)$ and $P_{2}(u)$ by different proportionality factors. On the contrary, there exists a unique function $\lambda(u)$ such that

$$
d\left(\lambda(u) x^{j}(u)\right) / d u=\lambda(u) \alpha(u) x_{1}^{j}(u) \quad(j=1,2,3) .
$$

For brevity we still use $x^{j}(u), x_{1}^{j}(u), x_{2}^{j}(u)$ instead of $\lambda(u) x^{j}(u), \lambda(u) x_{1}^{j}(u)$, $\lambda(u) x_{2}^{j}(u)$, so that (16) becomes

$$
d x^{j}(u) / d u=\alpha(u) x_{1}^{j}(u) .
$$

Through the independence of the three points $P, P_{1}, P_{2}, d x_{1}^{3}(u) / d u$ and $d x_{2}^{j}(u) / d u$ may be expressed in the form

$$
\begin{aligned}
& \frac{d x_{1}^{j}(u)}{d u}=\beta x^{j}(u)+\gamma x_{1}^{j}(u)+\delta x_{2}^{j}(u), \\
& \frac{d x_{2}^{j}(u)}{d u}=\mu x^{j}(u)+\nu x_{1}^{j}(u)+\tau x_{2}^{j}(u) .
\end{aligned}
$$

But the condition of immovability and (15) show that

Hence

$$
\begin{aligned}
\beta & =112 k(u) \alpha(u), & \gamma & =0, \\
\delta & =\alpha(u), & \mu & =5 \alpha(u) / 2, \\
\nu & =112 k(u) \alpha(u), & \tau & =0 .
\end{aligned}
$$

$$
\begin{gathered}
\frac{d x}{d u}=\alpha(u) x_{1}, \quad \frac{d x_{1}}{d u}=\alpha(u)\left(112 k(u) x+x_{2}\right), \\
\frac{d x_{2}}{d u}=\alpha(u)\left(\frac{5}{2} x+112 k(u) x_{1}\right),
\end{gathered}
$$

where we have written $x$ and $x_{i}(i=1,2)$ in place of $x^{j}$ and $x_{i}^{j}(j=1,2,3$; $i=1,2)$.

With respect to a parametric transformation $\bar{u}=f(u), \alpha(u) d u$ and $k(u)$ are clearly intrinsic.

A proper projective transformation

$$
x^{j}=\sum_{k=1}^{3} a_{k}^{j} y^{k} \quad(j=1,2,3),
$$

where $\left|a_{k}^{j}\right| \neq 0$, carries $x^{j}(u), x_{1}^{j}(u)$ and $x_{2}^{j}(u)(j=1,2,3)$ into the points $y^{j}(u), y_{1}^{j}(u)$ and $y_{2}^{j}(u)$ respectively. From the first two equations of (17), we find 


$$
\begin{aligned}
& \frac{d}{d u}\left(\sum_{k=1}^{3} a_{k}^{j} y^{k}\right)=\alpha(u)\left(\sum_{k=1}^{3} a_{k}^{j} y_{1}^{k}\right) \\
& \frac{d}{d u}\left(\sum_{k=1}^{3} a_{k}^{j} y^{k}\right)=\alpha(u)\left(112 k(u) \sum_{k=1}^{3} a_{k}^{j} y^{k}+\sum_{k=1}^{3} a_{k}^{j} y^{k}\right),
\end{aligned}
$$

or, because of $\left|a_{j}^{i}\right| \neq 0$,

$$
\frac{d y}{d u}=\alpha y_{1}, \quad \frac{d y_{1}}{d u}=\alpha\left(112 k(u) y+y_{2}\right) .
$$

That is, $\alpha d u$ and $k(u)$ are intrinsic and invariant. Consequently, we call them the projective arc-element and the projective curvature respectively.

Since any one of the points

$$
\left(\omega^{k}\left(\frac{d}{2 a^{4}}\right)^{1 / 3}, 1, \frac{\omega^{2 k}}{a^{2}}\left(\frac{d}{2 a^{4}}\right)^{2 / 3}\right) \quad(k=1,2,3)
$$

may be selected as the unit point, there are evidently three kinds of canonical coordinates. In consequence, the vertices of the fundamental triangle may have different canonical coordinates $z^{j}(u), z_{1}^{j}(u), z_{2}^{j}(u)$ and $x^{j}(u), x_{1}^{j}(u), x_{2}^{j}(u)$ $(j=1,2,3)$ respectively. They are, however, connected by

$$
x^{j}=z^{j} / \omega^{2}, \quad x_{1}^{j}=z_{1}^{j} / \omega, \quad x_{2}^{j}=z_{2}^{j} \quad(j=1,2,3),
$$

where $\omega^{3}=1, \omega \neq 1$.

The equations (17) then become

$$
\begin{gathered}
\frac{d z}{d u}=\alpha \omega z_{1}, \quad \frac{d z_{1}}{d u}=\alpha \omega\left(\frac{112 k(u)}{\omega^{2}} z+z_{2}\right), \\
\frac{d z_{2}}{d u}=\alpha \omega\left(\frac{5}{2} z+112 k(u) \cdot \frac{1}{\omega^{2}} z_{1}\right) .
\end{gathered}
$$

Therefore the projective arc-element and the projective curvature are multiplied by $\omega$ if another point among (19) is taken as the unit point.

If we put $d \sigma=\alpha d u,(17)$ reduces to the projective Frenet-Serret formulae required:

$$
\frac{d x}{d \sigma}=x_{1}, \quad \frac{d x_{1}}{d \sigma}=112 k(\sigma) x+x_{2}, \quad \frac{d x_{2}}{d \sigma}=\frac{5}{2} x+112 k(\sigma) x_{1} .
$$

Eliminating $x_{1}$ and $x_{2}$ from (A) gives

$$
-\frac{d^{3} x}{d \sigma^{3}}+224 k(\sigma) \frac{d x}{d \sigma}+\left(112 \frac{d k(\sigma)}{d \sigma}+\frac{5}{2}\right) x=0,
$$

which is preserved under the transformation 


$$
\bar{x}=\omega^{2} x, \quad \bar{k}(\sigma)=\omega k(\sigma), \quad d \bar{\sigma}=\omega d \sigma .
$$

Thus an analytic function $k(\sigma)(a \leqq \sigma \leqq b)$ corresponds except for a projectivity to an analytic curve, such that $d \sigma$ is the projective arc-element, $k(\sigma)$ the projective curvature, and independent solutions of the differential equation (B) the canonical coordinates of a current point of the curve. Conversely, an analytic curve corresponds to a unique projective curvature and a unique projective arc-element.

1.5. Geometrical interpretation of the projective curvature and the projective arc-element. By use of the unit point $I$ we can obtain the following interpretations $\left({ }^{3}\right)$ of the invariants appearing in (A):

$$
x_{1}\left(x, x_{2} ; d x_{1} / d \sigma, I\right)=112 k, \quad x_{2}\left(x, x_{1} ; x+d x, I\right)=d \sigma+(2),
$$

where the left members are cross ratios of the indicated pencils and (2) denotes an infinitesimal of higher order.

2. A generalization of sextactic points. In a recent paper [2] we have represented the neighborhood of various orders of a plane curve $C$ at its sextactic point $P$. A cusped quartic $Q_{4}$ is utilized such that its cusp lies on the tangent of $C$ at $P$ and its cuspidal tangent is also tangent to the hyperosculating conic of $C$ at $P$. Since either the sextactic point or its generalization appear in the theory of curves in five-dimensional space, we find it convenient to give here an outline of the theory on the generalized sextactic points.

2.1. The canonical expansion of a plane curve at its sextactic point. At a sextactic point $P(0,0,1)$ of a plane curve $C$ the osculating conic has a contact with the curve of order five. Hereafter we select the triangle of reference $\left\{P P_{1} P_{2}\right\}$ in such a way that this osculating conic is tangent to $P P_{1}$ and $P_{1} P_{2}$ at $P$ and $P_{2}$ respectively, so that the expansion of $\Gamma$ becomes

$$
y=a x^{2}+e x^{6}+f x^{7}+g x^{8}+h x^{9}+(10),
$$

and the equation of the osculating conic is $y-a x^{2}=0$.

The quartic $Q_{4}$, which has at $P$ a contact of order six with (20), is easily found to be

$$
\begin{aligned}
D y^{4}+E y^{3}(\alpha y-2 a x)- & \frac{8 e}{a \alpha} y^{3}\left(2 a x-\frac{2 a}{\alpha}-\frac{\alpha y}{2}\right) \\
& +\left(2 a x-\frac{2 a}{\alpha}-\frac{\alpha y}{2}\right)^{2}\left(4 a^{2} x^{2}-4 a y\right)=0,
\end{aligned}
$$

provided that $Q_{4}$ has a cusp at $A(1,0, \alpha)$. The polar line $l$ of $A$ with respect to the conic $y-a x^{2}=0$ intersects it again at $B\left(2 / \alpha, 4 a / \alpha^{2}\right)$. If $Q_{4}$ passes through $B$, the locus of the common points between $Q_{4}$ and $l$ consists of the conic and a covariant quartic

$\left(^{3}\right)$ For other interpretations see F. Vychyclo $[13,14]$. 


$$
-e y^{4} / a^{4}+\left(a x^{2}-y\right)^{2}=0 .
$$

The latter is particularly useful in determining the unit point.

When $Q_{4}$ given by (21) has at $P$ a contact of order seven with (20), then

$$
E=\left(8 e / a \alpha-8 f / a \alpha^{2}\right) .
$$

Furthermore, we can show that the join of the two common points, beside $P$, of $Q_{4}$ and the osculating conic passes through a fixed point $A^{\prime}[-e, 0,-2 a e+f]$. The correspondence between $A$ and $A^{\prime}$ is a projectivity with unique double point $H(e, 0, f)$, namely, the Halphen point discovered by Lane [8]. When it is taken for $(1,0,0)$, we have $f=0$. We select further the unit point on the osculating conic and $(1,1,0)$ on $(22)$, so that

$$
a=1, \quad e=1 \text {, }
$$

and the canonical expansion of the plane curve at its sextactic point becomes

$$
y=x^{2}+x^{6}+(8) \text {. }
$$

It should be noted that the selection of the unit point so as to satisfy (23) is not unique, but one of the three points $(-1,1),(i,-1)$ and $(-i,-1)$ can be taken for the unit point without changing the form (24).

2.2. The canonical expansion of a plane curve at a generalized sextactic point. A point $P$ on a curve $C$ is called a $k$-ic point if the osculating conic has at $P$ a $k$-point conic with the curve $C(k \geqq 6)$. Neither Lane's method nor ours in the foregoing section can be utilized in this case. We shall now determine the canonical expansions of a plane curve at a 7-ic or 8-ic point.

(i) The 7-ic point. A necessary and sufficient condition that $P$ is a 7-ic point is $a f \neq 0$ and $e=0$. If the quartic (21) has at $P$ a contact of order eight with (20), the equation of the quartic (21) takes the form

$$
\begin{aligned}
\left(-\frac{6 f \alpha}{a}+\frac{4 g}{a}\right) y^{4} & -\frac{8 f}{a} y^{3}(\alpha y-2 a x) \\
& +4 a\left(a x^{2}-y\right)\left(2 a \alpha x-2 a-\frac{\alpha^{2} y}{2}\right)^{2}=0 .
\end{aligned}
$$

The polar line of $A$ with respect to the osculating conic of $C$ at $P$ intersects $\left(21^{\prime}\right)$ at three points, and when $\alpha$ varies, one of them describes the curve

$$
\left(\frac{-12 f}{a^{2}} x y+\frac{4 g}{a^{3}} y^{2}\right) y^{4}+4 a\left(a x^{2}-y\right)^{3}=0 .
$$

This curve intersects the osculating conic in two different points on the line

$$
-3 a f x+g y=0 .
$$


Evidently, $g$ reduces to zero if $(26)$ is taken for the side $x=0$ of the triangle of reference.

In order that the unit point be on the osculating conic and $(1,1,0)$ on the curve (25), it is necessary and sufficient that

$$
a=1, \quad f=-2 / 3 \text {. }
$$

The canonical expansion of the curve $C$ then becomes

$$
y=x^{2}-2 x^{7} / 3+(9) \text {. }
$$

It is worth noticing that any one of the four points

$$
\left((1 / 2)^{1 / 5} e^{2 k \pi i / 5},(1 / 4)^{1 / 5} e^{4 k \pi i / 5}\right)
$$$$
(k=1,2,3,4)
$$

may be used for the unit point without changing the form (28).

(ii) The 8-ic point. If $P$ is an 8 -ic point, then

$$
\text { ag } \neq 0, \quad e=0, \quad f=0 .
$$

Neither the Halphen point nor the covariant line (26) can be determined. We require a further modification in the derivation of the canonical expansion.

In this case $\left(21^{\prime}\right)$ becomes

$$
4 g y^{4} / a+4 a\left(a x^{2}-y\right)\left(2 a \alpha x-2 a-\alpha^{2} y / 2\right)^{2}=0,
$$

which has at $P$ a contact of order nine with $C$ if

$$
\alpha=h / 2 g \text {. }
$$

Thus we arrive at a covariant point $(2 g, 0, h)$. Upon selecting $S$ for $(1,0,0)$, $\alpha$ and $h$ reduce to zero and the corresponding 10-point quartic of $S$ is found to be

$$
g y^{4} / a+a^{3}\left(a x^{2}-y\right)=0 .
$$

If the common points of $x=0$ and (30) are projected from $S$ to the osculating conic, we obtain six points of coordinates

$$
\left( \pm(1 / a)^{1 / 2}\left(g / a^{4}\right)^{1 / 6} \omega^{k / 2}, 1,\left(g / a^{4}\right)^{1 / 3} \omega^{k}\right) \quad\left(k=1,2,3, \omega^{3}=1, \omega \neq 1\right) .
$$

By choosing any one of them for the unit point both $a$ and $g$ reduce to 1 , so that the canonical expansion of $C$ in the neighborhood of $P$ is found to be

$$
y=x^{2}+x^{8}+(10) \text {. }
$$

Chapter II. The projective differential theory of CURves IN FIVE-DIMENSIONAL SPACE

In $\$ 3$ we construct a covariant pyramid of reference at an ordinary point of a curve in five-dimensional space. In addition to some simple interpretations of the invariants of the curve the canonical expansions of five different types are given. 
3. The covariant pyramid. Suppose that the point $P=P(u)$ describes a curve $\Gamma$ in five-dimensional space where $u$ is a parameter. Take $\left\{P P_{1} P_{2} P_{3} P_{4} P_{5}\right\}$ for the local pyramid of reference associated with a generic point $P$ of $\Gamma$, such that $P_{j}$ lies in the osculating linear space $S_{j}(P)$ of $j$ dimensions at $P$ to $\Gamma$, but not in the osculating space $S_{j-1}(P)$. Any point $Q$ in the space can be expressed by $\sum_{\tau=0}^{\mathrm{s}} X_{\tau} P_{r}$, where $P_{0} \equiv P$.

The nonhomogeneous coordinates are defined by putting

$$
x_{1}=X_{1} / X_{0}, x_{2}=X_{2} / X_{0}, \cdots, x_{5}=X_{5} / X_{0} .
$$

In case the parametric equation of the curve is of the form

$$
X(u)=\sum_{r=0}^{5} X_{r}(u) P_{r}
$$

the expansions of the nonhomogeneous coordinates of $\Gamma$ are

$$
x_{i}=\sum_{j=0}^{\infty} b_{i, i+j}\left(x_{1}\right)^{i+j}
$$$$
\left(\prod_{2}^{5} b_{i, i} \neq 0, i=2,3,4,5\right)
$$

where $b_{i, i+j}$ are local functions.

3.1. The developable hypersurface of $\Gamma$. Since $x_{1}$ depends upon $u$, we may regard $x_{1}, \lambda_{1}, \lambda_{2}$ and $\lambda_{3}$ as parameters and obtain the parametric equations of the developable hypersurface of $\Gamma$ :

$$
\begin{aligned}
& X_{0}=1, \quad X_{1}=x_{1}+\lambda_{1}, \\
& X_{k}=x_{k}+\sum_{v=1}^{3} \lambda_{\nu} \frac{d^{v} x^{k}}{\left(d x_{1}\right)^{\nu}} \quad(k=2,3,4,5) .
\end{aligned}
$$

The section $C$ of this hypersurface produced by the osculating plane of $\Gamma$ at its ordinary point $P$ is given by the equations

$$
\begin{array}{ll}
X_{0}=D\left[d x_{3} / d x_{1}, d x_{4} / d x_{1}, d x_{5} / d x_{1}\right], & X_{1}=D\left[x_{1}, x_{3}, x_{4}, x_{5}\right], \\
X_{2}=D\left[x_{2}, x_{3}, x_{4}, x_{5}\right], \quad X_{3}=0, & X_{4}=0, \quad X_{6}=0,
\end{array}
$$

where $D[\cdots]$ denotes the Wronskian.

Putting, for brevity,

$$
\xi_{0}=\frac{X_{0}}{x_{1}^{6}}=\sum_{0}^{\infty} B_{\nu} x_{1}^{\nu}, \quad \xi_{1}=\frac{X_{1}}{x_{1}^{6}}=x_{1} \sum_{0}^{\infty} C_{\nu} x_{1}^{\nu}, \quad \xi_{2}=\frac{X_{2}}{x_{1}^{6}}=x_{1}^{2} \sum_{0}^{\infty} D_{\nu} x_{1}^{\nu},
$$

we find that if $P_{2}$ lies on the osculating conic $C_{2}$ of $C$ and if $P_{1} P_{2}$ is the tangent to $C_{2}$, then

$$
\begin{aligned}
2 D_{0} B_{0} C_{1}-C_{0} D_{0} B_{1}-C_{0} D_{1} B_{0} & =0 \\
D_{0} B_{0}\left(C_{1}^{2}+2 C_{0} C_{2}\right)-C_{0}^{2} B_{2} D_{0}-D_{1} B_{1} C_{0}^{2}-B_{0} D_{2} C_{0}^{2} & =0 .
\end{aligned}
$$

Consequently, the osculating conic of $\Gamma$ at $P$ is given by the equations 
(6)

$$
B_{0} D_{0} X_{1}^{2} / C_{0}^{2}-X_{0} X_{2}=0, \quad X_{3}=0, \quad X_{4}=0, \quad X_{5}=0 .
$$

A simple calculation gives

$$
\begin{array}{ll}
B_{0}=120 \prod_{3}^{5} b_{i, i}, & C_{0}=48 \prod_{3}^{5} b_{i, i} \\
D_{0}=12 \prod_{2}^{5} b_{i, i}, & B_{1}=2 \cdot 6^{8} \cdot\left(\prod_{3}^{5} b_{i, i}\right) \cdot \frac{b_{5,6}}{b_{5,5}} \\
C_{1}=180\left(\prod_{3}^{5} b_{i, i}\right) \frac{b_{5,6}}{b_{5,5}}, & D_{1}=48\left(\prod_{2}^{5} b_{i, i}\right) \cdot \frac{b_{5,6}}{b_{5,5}}, \\
B_{2}=-3^{3} \cdot 20 b_{3,3} b_{4,6} b_{5,5}+7 \cdot 144 b_{3,3} b_{4,4} b_{5,7}, \\
C_{2}=-240 b_{3,3} b_{4,6} b_{5,5}+3^{3} \cdot 2^{4} b_{3,3} b_{4,4} b_{5,7} \\
D_{2}=-72 b_{2,2} b_{3,3} b_{4,6} b_{5,5}+120 b_{2,2} b_{3,3} b_{4,4} b_{5,7} .
\end{array}
$$

Substituting them in (5) and (6), we have

$$
b_{5,6}=0 \text {, }
$$

and

$$
5 b_{5,6} b_{4,6}-4 b_{4,4} b_{5,7}=0 .
$$

When $P$ is an ordinary point of $C$, we define the fundamental triangle $\left\{P P_{1} P_{2}\right\}$ by the method stated in $\$ 1.1$. The cubic,

$$
\left(\frac{D_{0} B_{0}}{C_{0}^{2}} X_{1}^{2}-X_{0} X_{2}\right) X_{0}-X_{1} X_{2}^{2}\left(\frac{2 C_{3} B_{0}^{2}}{C_{0}^{2} D_{0}}-\frac{B_{0}^{2} D_{3}}{C_{0} D_{0}^{2}}-\frac{B_{0} B_{3}}{C_{0} D_{0}}\right)=0,
$$

which is determined by the conditions: (i) it passes through $P_{1}$; (ii) it is tangent to (6) at $P_{2}$; and (iii) it has at $P$ a contact of order five with $C$, should have at $P$ a contact of order six with $C$. In other words,

$$
\left(D_{0} B_{0} / C_{0}^{2}\right)\left(C_{2}^{2}+2 C_{0} C_{4}\right)-D_{0} B_{4}-D_{4} B_{0}-D_{2} B_{2}=0 .
$$

Otherwise, for $A \neq 0$,

$$
\left(\frac{D_{0} B_{0}}{C_{0}^{2}} X_{1}^{2}-X_{0} X_{2}\right) X_{1}-X_{1} X_{2}^{2}\left(\frac{2 C_{3} B_{0}{ }^{2}}{C_{0}^{2} D_{0}}-\frac{B_{0} D_{3}^{2}}{C_{0} D_{0}^{2}}-\frac{B_{0} B_{3}}{C_{0} D_{0}}\right)+A X_{2}^{3}=0
$$

would have at $P$ a contact of order six with $C$, which contradicts the hypothesis that $P_{1} P_{2}$ does not pass through any point common to (12) and the osculating conic.

3.2. The construction of the quadric $Q_{3}$. A plane passing through $P P_{1}$ and lying in the osculating three-space of $\Gamma$ at $P$ is given by the equations

$$
X_{3}-\mu X_{2}=0, \quad X_{4}=0, \quad X_{5}=0 .
$$


If the osculating planes of $\Gamma$ be projected from a point $(1,0, \rho, 0,0,0)$ on the above plane, we obtain a plane curve $\Gamma_{1}$, whose equations are easily found to be (13) and

$$
\begin{aligned}
& X_{1}=\frac{1}{x_{1}^{6}} D\left[x_{1}, x_{4}, x_{5}\right], \quad X_{3}=\frac{1}{x_{1}^{6}} D\left[x_{3}, x_{4}, x_{5}\right] \\
& X_{0}=\frac{1}{x_{1}^{6}} D\left[\frac{d x_{4}}{d x_{1}}, \frac{d x_{5}}{d x_{1}}\right]+\frac{1}{\rho x_{1}^{6}} D\left[x_{4}, x_{5}, \frac{1}{\mu} x_{3}-x_{2}\right]
\end{aligned}
$$

Put

$$
\begin{aligned}
& X_{1}=x_{1} \sum_{0}^{\infty} E_{\nu} x_{1}^{\prime}, \quad X_{3}=x_{1}^{3} \sum_{0}^{\infty} F_{\nu} x_{1}^{\prime}, \\
& X_{0}=\sum_{0}^{\infty} G_{\nu} x_{1}^{\prime}+\frac{1}{\rho \mu} x_{1}^{3} \sum_{0}^{\infty} F_{\nu} x_{1}^{\nu}-\frac{x_{1}^{2}}{\rho} \sum_{0}^{\infty} H_{\nu} x_{1}^{\prime} .
\end{aligned}
$$

Then

$$
\begin{aligned}
& E_{\nu}=\sum_{\nu_{1}+\nu_{2}=\nu} b_{4,4+\nu_{1}} b_{5,5+\nu_{2}}\left(3+\nu_{1}\right)\left(4+\nu_{2}\right)\left(1+\nu_{2}-\nu_{1}\right), \\
& F_{\nu}=\sum_{\nu_{1}+\nu_{2}+\nu_{3}=\nu} b_{3,3+\nu_{1}} b_{4,4+\nu_{2}} b_{5,5+\nu_{3}} P\left(\nu_{1}, 1+\nu_{2}, 2+\nu_{3}\right), \\
& G_{\nu}=\sum_{\nu_{1}+\nu_{2}=\nu}\left(4+\nu_{1}\right)\left(5+\nu_{2}\right) b_{4,4+\nu_{1}} b_{5,5+\nu_{2}}\left(1+\nu_{2}-\nu_{1}\right), \\
& H_{\nu}=\sum_{\nu_{1}+\nu_{2}+\nu_{3}=\nu}-b_{2,2+\nu_{1}} b_{5,5+\nu_{2}} b_{4,4+\nu_{3}} P\left(\nu_{1}, 2+\nu_{3}, 3+\nu_{2}\right)
\end{aligned}
$$

where

$$
P\left(\mu_{1}, \mu_{2}, \cdots, \mu_{n}\right)=\prod_{i j \geq 1}^{n}\left(\mu_{i}-\mu_{j}\right) .
$$

For the subsequent discussion it is convenient to give here an outline of the theory of singularities of a plane curve $[5,6]$. If the expansions of the coordinates of a plane curve are

$$
x=s \sum_{0}^{\infty} a_{\nu} s^{\nu}, \quad y=s^{m} \sum_{0}^{\infty} b_{\nu} s^{\nu}, \quad z=1+\sum_{1}^{\infty} c_{\nu} s^{\nu},
$$

the point $(0,0,1)$ is a singular point of the curve denoted by $S_{1}{ }^{m}$. When $(m-3)$ conditions are satisfied, this singularity is particularly simple. Let $U_{10, k}$ and $A_{m, k}$ be respectively the coefficients of $s^{k}$ in the expansions of $\left(\sum_{0}^{\infty} b_{\nu} s^{\nu}\right)$ $\cdot\left(1+\sum_{1}^{\infty} c_{\nu} s^{\nu}\right)^{m-1}$ and $\left(\sum_{0}^{\infty} a_{\nu} s^{\nu}\right)^{m}$. If

$$
A_{m, 1}-a_{0}^{m} U_{10,1} / b_{0}=0,
$$

the coordinates of the covariant points $O_{m+1}$ and $O_{2 m}$ of the curve are $(1,0,0)$ and 


$$
\left(\frac{1}{m a_{0}^{m-1} b_{0}}\left(A_{m, m-1}-\frac{a_{0}^{m}}{b_{0}} U_{10, m-1}\right), 1, \frac{1}{a_{0}^{m} b_{0}(m-1)}\left(\frac{a_{0}^{m}}{b_{0}} U_{10, m}-A_{m, m}\right)\right) .
$$

Moreover, the conditions of representability become

$$
a_{0}^{m} U_{10, k} / b_{0}-A_{m, k}=0 \quad(k=2, \cdots, m-2) .
$$

From (8) it follows that the osculant $O_{4}$ associated with the point of inflexion $P$ of $\Gamma_{1}$ always coincides with $P_{1}$. The coordinates of $O_{6}$ are

$$
\begin{aligned}
& X_{4}=0, \quad X_{6}=0, \quad X_{3}=1, \quad X_{2}=1 / \mu, \\
& X_{1}=-\frac{1}{3}\left\{\frac{E_{0} F_{2}}{F_{0}^{2}}+\frac{2 E_{0} G_{2}}{F_{0} G_{0}}-3 \frac{F_{2}}{F_{0}}-2 \frac{E_{0} H_{0}}{\rho F_{0} G_{0}}\right\}, \\
& X_{0}=\frac{G_{0}^{2}}{2 E_{0} F_{0}}\left\{\frac{E_{0}}{F_{0}}\left(\frac{F_{3}}{G_{0}}+\frac{2 F_{0} G_{3}}{G_{0}^{2}}-\frac{2 F_{0} H_{1}}{\rho G_{0}^{2}}+2 \frac{E_{0} F_{0}}{G_{0}^{2} \rho \mu}\right)-3 \frac{E_{3}}{G_{0}}\right\} .
\end{aligned}
$$

Eliminating $\mu$ we obtain $\left(^{4}\right)$ a generator of the quadric $Q_{3}$ :

$$
\begin{gathered}
\frac{X_{0}}{X_{3}}=\frac{1}{2}\left(\frac{G_{0} F_{3}}{F_{0}^{2}}+2 \frac{G_{3}}{F_{0}}-3 \frac{G_{0} E_{3}}{E_{0} F_{0}}\right) \\
+\frac{F_{0} G_{0}}{2 E_{0} H_{0}}\left(\frac{X_{2}}{X_{3}}-\frac{H_{1}}{F_{1}}\right)\left(\frac{3 X_{1}}{X_{3}}+\frac{E_{0} F_{2}}{F_{0}^{2}}+2 \frac{E_{0} G_{2}}{F_{0} G_{0}}-3 \frac{E_{2}}{F_{0}}\right), \\
X_{4}=0, \quad X_{5}=0 .
\end{gathered}
$$

Since $P, P_{1}$ and $P_{2}$ lie on the same quadrilateral of the quadric, we take the fourth vertex as $P_{3}$. Then

$$
\begin{array}{cl}
G_{0} E_{0} F_{3}+2 E_{0} F_{0} G_{3}-3 G_{0} E_{3} F_{0}=0, & H_{1}=0, \\
E_{0} F_{2} G_{0}+2 E_{0} F_{0} G_{2}-3 G_{0} F_{0} E_{2}= & 0 .
\end{array}
$$

It is easily verified by means of (8) that

$$
\begin{array}{cl}
E_{0}=12 b_{4,4} b_{5,5}, & F_{0}=2 b_{3,3} b_{4,4} b_{5,5}, \\
G_{0}=20 b_{4,4} b_{5,5}, \quad H_{1}=3 b_{2,3}, & E_{2}=-20 b_{4,6} b_{5,5}+54 b_{4,4} b_{5,7} \\
F_{2}=-6 b_{3,3} b_{4,6} b_{5,5}+12 b_{3,3} b_{4,4} b_{5,7}, & G_{2}=-30 b_{5,5} b_{4,6}+84 b_{4,4} b_{5,7}
\end{array}
$$

Hence the last two equations of (19) become

$$
\begin{gathered}
b_{2,3}=0, \\
-310 b_{5,5} b_{4,6}+729 b_{4,4} b_{5,7}=0 .
\end{gathered}
$$

Combining (19) and (21) we find

(4) This is a result analogous to what we obtained in 1942 in studying the point of inflexion of a space curve, cf. Chang [3]. 


$$
b_{4,6}=0, \quad b_{5,7}=0
$$

and consequently

$$
\begin{gathered}
F_{3}=2 b_{3,6} b_{4,4} b_{5,5}-16 b_{3,3} b_{4,7} b_{5,5}+20 b_{3,3} b_{4,4} b_{5,8} \\
G_{3}=-70 b_{5,5} b_{4,7}+128 b_{4,4} b_{5,8}, \quad E_{3}=-48 b_{5,5} b_{4,7}+48 b_{4,4} b_{5,8}
\end{gathered}
$$

Therefore the first equation of (19) becomes

$$
b_{3,6} / b_{3,3}-3 b_{4,7} / b_{4,4}+9 b_{5,8} / 5 b_{5,5}=0 .
$$

The covariant quadric (18) is now given by the equations

$$
X_{4}=0, \quad X_{5}=0, \quad X_{0} X_{3}=\left(5 b_{3,3} / 6 b_{2,2}\right) X_{1} X_{2} .
$$

3.3. The construction of the quadric $Q_{4}$. In a similar way we proceed to determine the vertex $P_{4}$ of the pyramid $\left\{P, P_{1}, \cdots, P_{5}\right\}$. In fact, let the osculating planes of $\Gamma$ be projected from $(1,0,0, \rho, 0,0)$ to a plane passing through $P P_{1}$ and lying in the osculating space of four dimensions of $\Gamma$ at $P$ :

$$
X_{5}=0, \quad X_{2}-\mu X_{4}=0, \quad X_{3}-\nu X_{4}=0 .
$$

The equations of the plane curve $\Gamma_{2}$ thus produced can be obtained by eliminating $\lambda_{1}, \lambda_{2}$ and $\lambda_{3}$ from (25) and

$$
\begin{aligned}
& X_{0}=1+\lambda_{3}, \quad X_{1}=x_{1}+\lambda_{1}, \\
& X_{k}=x_{k}+\lambda_{1} d x_{k} / d x_{1}+\lambda_{2} d^{2} x_{k} /\left(d x_{1}\right)^{2} \\
& X_{3}=x_{3}+\lambda_{1} d x_{3} / d x_{1}+\lambda_{2} d^{2} x_{3} /\left(d x_{1}\right)^{2}+\lambda_{3} \rho .
\end{aligned} \quad(k=2,4,5),
$$

The result of carrying out the computation shows that $\Gamma_{2}$ is given by (25) and

$$
\begin{aligned}
& X_{0}=\frac{1}{x_{1}^{4}}\left\{D\left[\frac{d x_{5}}{d x_{1}}, \frac{d\left(x_{2}-\mu x_{4}\right)}{d x_{1}}\right]-\frac{1}{\rho} D\left[x_{5}, x_{2}-\mu x_{4}, x_{3}-\nu x_{4}\right]\right\}, \\
& X_{1}=\frac{1}{x_{1}^{4}}\left\{x_{1} D\left[\frac{d x_{5}}{d x_{1}}, \frac{d\left(x_{2}-\mu x_{4}\right)}{d x_{1}}\right]-\frac{d}{d x_{1}} D\left[x_{5}, x_{2}-\mu x_{4}\right]\right\}, \\
& X_{4}=D\left[x_{2}, x_{4}, x_{5}\right] .
\end{aligned}
$$

For brevity, we put

$$
\begin{aligned}
& D\left[\frac{d x_{5}}{d x_{1}}, \frac{d x_{2}}{d x_{1}}\right]=x_{1}^{4} \sum_{0}^{\infty} E_{\tau}^{\prime} x_{1}^{\tau}, \\
& D\left[\frac{d x_{5}}{d x_{1}}, \frac{d x_{4}}{d x_{1}}\right]=x_{1}^{6} \sum_{0}^{\infty} F_{\tau}^{\prime} x_{1}^{\top}, \\
& \frac{d}{d x_{1}} D\left[x_{5}, x_{2}\right]=x_{1}^{5} \sum_{0}^{\infty}(6+\tau) G_{\tau}{ }^{\prime} x_{1}{ }^{\tau} \text {, } \\
& \frac{d}{d x_{1}} D\left[x_{5}, x_{4}\right]=x_{1}^{7} \sum_{0}^{\infty} H_{\tau}^{\prime} x_{1}^{r} \text {, } \\
& D\left[x_{5}, x_{3}, x_{2}\right]=x_{1}^{7} \sum_{0}^{\infty} I_{\tau} x_{1}^{\tau},
\end{aligned}
$$


so that (26) reduces to

$$
\begin{aligned}
& X_{0}=\sum_{0}^{\infty} E_{\tau}^{\prime} x_{1}^{\tau}-\mu x_{1}^{2} \sum_{0}^{\infty} F_{\tau}^{\prime} x_{1}^{\tau}-\frac{x_{1}^{3}}{\rho} \sum_{0}^{\infty} I_{\tau} x_{1}^{\tau} \\
& \quad+\frac{\nu x_{1}}{\rho} \sum_{0}^{\infty} H_{\tau} x_{1}^{\tau}-\frac{\mu}{\rho} x_{1}^{5} \sum_{0}^{\infty} F_{\nu} x_{1}^{\tau}, \\
& X_{1}=x_{1} \sum_{0}^{\infty} E_{\tau}^{\prime} x_{1}^{\tau}-x_{1} \sum_{0}^{\infty} G_{\tau}^{\prime}(\tau+6) x_{i}^{\tau}+\mu x_{1}^{3} \sum_{0}^{\infty}\left[(\tau+8) H_{\tau}^{\prime}-F_{\tau}^{\prime}\right] x_{1}^{\tau}, \\
& X_{4}=x_{1}^{4} \sum_{0}^{\infty} H_{\tau} x_{1}^{\tau} .
\end{aligned}
$$

The point $P$ is evidently a singularity [5] $S_{1}^{4}$ of $\Gamma_{2}$. Since the osculant $Q_{5}$ always coincides with $P_{1}$, the singular point $S_{1}^{4}$ of $\Gamma_{2}$ is representable when and only when

$$
\frac{E_{0}^{\prime}-6 G_{0}^{\prime}}{E_{0}^{\prime}}\left(\frac{H_{2}}{H_{0}}+3 \frac{E_{2}^{\prime}-\mu F_{2}^{\prime}}{E_{0}^{\prime}}\right)-4 \frac{E_{2}^{\prime}-8 G_{2}^{\prime}}{E_{0}^{\prime}}-4 \mu \frac{8 H_{0}^{\prime}-F_{0}^{\prime}}{E_{0}^{\prime}}=0 .
$$

Therefore $\Gamma_{2}$ has a representable singular point at $P$ if and only if the plane (25) belongs to the three-dimensional space $X_{5}=0, X_{2}-\mu X_{4}=0$, where $\mu$ is given by (28). To select $P_{4}$ in this space brings $\mu$ to zero, and (28) to

$$
\frac{E_{0}^{\prime}-6 G_{0}^{\prime}}{E_{0}^{\prime}}\left(\frac{H_{2}}{H_{0}}+3 \frac{E_{2}^{\prime}-\mu F_{2}^{\prime}}{E_{0}^{\prime}}\right)-4 \frac{E_{2}^{\prime}-8 G_{2}^{\prime}}{E_{0}^{\prime}}=0 \text {. }
$$

A simple calculation of Wronskians shows that

$$
\begin{array}{rlrl}
E_{0}{ }^{\prime} & =-30 b_{5,5} b_{2,2}, & F_{0}{ }^{\prime}=-20 b_{5,5} b_{4,4}, & G_{0}{ }^{\prime}=-3 b_{2,2} b_{5,5}, \\
H_{0}^{\prime}=-b_{5,5} b_{4,4}, & H_{0}=6 b_{2,2} b_{4,4} b_{5,5}, & I_{0}=6 b_{2,2} b_{3,3} b_{5,5}, \\
H_{2}=0, & E_{2}^{\prime}=-20 b_{4,4} b_{5,5}, & G_{2}{ }^{\prime}=-b_{2,4} b_{5,5} .
\end{array}
$$

Hence (28) becomes

$$
b_{2,4}=0 .
$$

The coordinates of the covariant point $O_{8}$ are easily found to be

$$
\begin{aligned}
& X_{5}=0, \quad X_{4}=1, \quad X_{3}=\nu, \\
& X_{1}=\frac{-E_{0}^{\prime}}{4 H_{0}}\left[\left(\frac{E_{0}^{\prime}-6 G_{0}^{\prime}}{E_{0}^{\prime}}\right)\left(\frac{H_{3}}{H_{0}}-4 \frac{E_{3}^{\prime}}{E_{0}^{\prime}}\right)+3 \frac{E_{3}^{\prime}-9 G_{3}^{\prime}}{E_{0}^{\prime}}\right] \\
& \quad+\frac{3 I_{0}}{4 \rho H_{0}}\left(\frac{E_{0}^{\prime}-6 G_{0}^{\prime}}{E_{0}^{\prime}}\right), \\
& X_{0}=\frac{1}{3}\left[\frac{E_{0}^{\prime} H_{4}}{H_{0}^{2}}+\frac{3 E_{4}^{\prime}}{H_{0}}-\frac{4 E_{0}^{\prime}}{H_{0}}\left(\frac{E_{4}^{\prime}-10 G_{4}^{\prime}}{E_{0}^{\prime}-6 G_{0}^{\prime}}\right)\right]+\frac{1}{\rho H_{0}}\left(-I_{1}+\nu H_{0}\right),
\end{aligned}
$$


whose locus is a quadric $Q_{4}$ :

$$
\begin{aligned}
& \frac{X_{0}}{X_{4}}=\frac{1}{3} {\left[-\frac{E_{0}^{\prime} H_{4}}{H_{0}^{2}}+\frac{3 E_{4}^{\prime}}{H_{0}}-\frac{E_{4}^{\prime}-10 G_{4}^{\prime}}{10 H_{0}}\right] } \\
&+ \frac{4}{3}\left(\frac{E_{0}^{\prime}}{E_{0}^{\prime}-6 G_{0}^{\prime}}\right)\left(-\frac{I_{1}}{I_{0}}+\frac{H_{0}}{I_{0}} \frac{X_{3}}{X_{4}}\right) \\
& \cdot\left[\frac{X_{1}}{X_{4}}+\frac{E_{0}^{\prime}}{G_{0}^{\prime}}\left(\frac{E_{0}^{\prime}-6 G_{0}^{\prime}}{E_{0}^{\prime}}\right)\left(\frac{H_{3}}{H_{0}}+\frac{3 E_{3}^{\prime}}{E_{0}^{\prime}}\right)-4 \frac{E_{3}^{\prime}-6 G_{3}^{\prime}}{6 H_{0}}\right], \\
& X_{5}=0, \quad X_{2}=0 .
\end{aligned}
$$

In order that $\left\{P_{0} P_{1} P_{8} P_{4}\right\}$ be a quadrilateral on $Q_{4}$, it is necessary and sufficient that

$$
I_{1}=0 \text {, }
$$

$$
\begin{array}{r}
\left(\left(E_{0}^{\prime}-6 G_{0}^{\prime}\right) / E_{0}^{\prime}\right)\left(H_{3} / H_{0}+3\left(E_{3}^{\prime} / E_{0}^{\prime}\right)\right)-4\left(E_{3}^{\prime}-6 G_{3}^{\prime}\right) / E_{0}^{\prime}=0 \\
E_{0}^{\prime} H_{4} / H_{0}^{2}+3 E_{4}^{\prime} / H_{0}-10\left(E_{4}^{\prime}-10 G_{4}^{\prime}\right) / H_{0}=0
\end{array}
$$

or

$$
\begin{gathered}
b_{3,4}=0 \\
25 b_{4,7} / b_{4,4}-18 b_{5,8} / b_{5,5}=0 \\
-20 b_{2,6} / b_{2,2}+45 b_{4,8} / b_{4,4}-28 b_{5,9} / b_{5,5}=0
\end{gathered}
$$

because of (8), (20), (22), (23), (29), (34) and

Putting$$
E_{3}{ }^{\prime}=96 b_{2,2} b_{5,8}, \quad G_{3}{ }^{\prime}=-6 b_{2,2} b_{5,8},
$$$$
H_{3}=\left(-30 b_{4,7} / b_{4,4}+48 b_{5,8} / b_{5,5}\right) b_{2,2} b_{4,4} b_{5,5}
$$$$
H_{4}=\left(2 b_{2,8} / b_{2,2}-54 b_{4,8} / b_{4,4}+70 b_{5,9} / b_{5,6}\right) b_{2,2} b_{4,4} b_{5,5}
$$$$
E_{4}^{\prime}=\left(-126 b_{5,9} / b_{5,5}+30 b_{2,6} / b_{2,2}\right) b_{2,2} b_{5,5}
$$$$
G_{4}^{\prime}=\left(-7 b_{5,9} / b_{5,5}+b_{2,6} / b_{2,2}\right) b_{2,2} b_{5,5} \text {. }
$$

$$
b_{4,7} / b_{4,4}=72 \omega_{1},
$$

we know by means of (23) and (34) that

$$
b_{6,8} / b_{6,5}=100 \omega_{1}, \quad b_{3,6} / b_{3,3}=36 \omega_{1} .
$$

3.4. The construction of the quadric $Q_{5}$. We come now to locate the remaining vertex $P_{5}$ of the covariant pyramid $\left\{P, P_{1}, P_{2}, P_{3}, P_{4}, P_{5}\right\}$. A plane $p$ passing through $P P_{1}$ is, in general, given by the equations

$$
X_{2}-\nu_{1} X_{5}=0, \quad X_{3}-\nu_{2} X_{5}=0, \quad X_{4}-\nu_{3} X_{5}=0 .
$$

If a three-dimensional space contains the point $(1,0,0,0, \rho, 0)$ and the osculating plane of $\Gamma$, then it must be given by the equations 


$$
\begin{gathered}
X_{0}=1+\lambda_{1}, \quad X_{1}=x_{1}+\lambda_{2}, \quad X_{4}=x_{4}+\sum_{i=1}^{2} \lambda_{v+1} \frac{d^{v} x_{4}}{\left(d x_{1}\right)^{v}}+\lambda_{1} \rho, \\
X_{k}=x_{k}+\sum_{v=1}^{2} \lambda_{v+1} \frac{d^{v} x_{k}}{\left(d x_{1}\right)^{v}} \quad(k=2,3,5),
\end{gathered}
$$

where $x_{1}, \lambda_{1}, \lambda_{2}$ and $\lambda_{3}$ are parameters. Eliminating $\lambda_{1}, \lambda_{2}$ and $\lambda_{3}$ from (37) and (38) we obtain a plane curve $\Gamma_{3}$, which is given by (37) and

$$
\begin{aligned}
X_{0}=\frac{1}{x_{1}^{2}}\left\{D \left[\frac{d}{d x_{1}}\left(x_{2}-\nu_{1} x_{5}\right),\right.\right. & \left.\frac{d}{d x_{1}}\left(x_{3}-\nu_{2} x_{5}\right)\right] \\
& \left.-\frac{1}{\rho} D\left[x_{2}-\nu_{1} x_{5}, x_{3}-\nu_{2} x_{5}, x_{4}-\nu_{3} x_{5}\right]\right\},
\end{aligned}
$$

$\left(39^{\prime}\right) X_{1}=\frac{1}{x_{1}^{2}}\left\{x_{1} D\left[\frac{d}{d x_{1}}\left(x_{2}-\nu_{1} x_{5}\right), \frac{d}{d x_{1}}\left(x_{3}-\nu_{2} x_{6}\right)\right]\right.$

$$
\left.-\frac{d}{d x_{1}} D\left[x_{2}-\nu_{1} x_{5}, x_{8}-\nu_{2} x_{5}\right]\right\}
$$

$$
X_{5}=\frac{1}{x_{1}^{2}} D\left[x_{5}, x_{2}, x_{3}\right]
$$

We put

$$
\begin{array}{rlrl}
D\left[\frac{d x_{2}}{d x_{1}}, \frac{d x_{3}}{d x_{1}}\right] & =x_{1}^{2} \sum_{0}^{\infty} I_{\nu}^{\prime} x_{1}^{\prime}, & D\left[\frac{d x_{5}}{d x_{1}}, \frac{d x_{3}}{d x_{1}}\right]=x_{1}^{5} \sum_{0}^{\infty} J_{v}^{\prime} x_{1}^{\prime}, \\
D\left[x_{2}, x_{3}, x_{4}\right]=x_{1}^{6} \sum_{0}^{\infty} J_{v} x_{1}^{\prime}, & \frac{d}{d x_{1}} D\left[x_{2}, x_{3}\right]=x_{1}^{8} \sum_{0}^{\infty} K_{v}^{\prime} x_{1}^{\prime},
\end{array}
$$

and substitute them in $\left(39^{\prime}\right)$, so that $\Gamma_{3}$ is given by (37) and

$$
\begin{aligned}
X_{5}= & x_{1}^{5} \sum_{0}^{\infty} I_{\nu} x_{1}^{\prime}, \\
X_{1}= & x_{1} \sum_{0}^{\infty}\left(I_{\nu}^{\prime}-K_{\nu}^{\prime}\right) x_{1}^{\prime}+\nu_{2} x_{1}^{8} \sum_{0}^{\infty}\left[E_{\nu}^{\prime}-G_{\nu}^{\prime}(\nu+6)\right] x_{1}^{\prime} \\
& +\nu_{1} x_{1}^{4} \sum_{0}^{\infty}\left(L_{\nu}^{\prime}-J_{\nu}^{\prime}\right) x_{1}^{\prime}, \\
X_{0}= & \sum_{0}^{\infty} I_{\nu}^{\prime} x_{1}^{\prime}+\nu_{2} x_{1}^{2} \sum_{0}^{\infty} E_{\nu}^{\prime} x_{1}^{\prime}-\nu_{1} x_{1}^{3} \sum_{0}^{\infty} J_{\nu}^{\prime} x_{1}^{\prime} \\
& \quad-\frac{x_{1}^{4}}{\rho} \sum_{0}^{\infty} J_{\nu} x_{1}^{\prime}+\frac{\nu_{3}}{\rho} x_{1}^{6} \sum_{0}^{\infty} I_{\nu} x_{1}^{\prime}-\frac{\nu_{2}}{\rho} x_{1}^{6} \sum_{0}^{\infty} H_{\nu} x_{1}^{\prime}+\frac{\nu_{1}}{\rho} x_{1}^{7} \sum_{0}^{\infty} F_{\nu} x_{1}^{\prime} .
\end{aligned}
$$

The curve $\Gamma_{8}$ has a singularity $S_{1}^{5}$ at $P$, whose covariant point $O_{6}$ also coin- 
cides with $P_{1}$. But the covariant points $O_{7, i}(i=1,2)[6]$ will coincide when and only when

$$
\begin{aligned}
\frac{a_{0}^{5}}{b_{0}} U_{10,2}-A_{5,2}= & \left.\frac{I_{0}^{\prime}-K_{0}^{\prime}}{I_{0}^{\prime}}\right)^{5}\left(\frac{I_{2}}{I_{0}}+4 \frac{I_{2}^{\prime}+E_{0}^{\prime} \nu_{2}}{I_{0}^{\prime}}\right) \\
& -5\left(\frac{I_{0}^{\prime}-K_{0}^{\prime}}{I_{0}^{\prime}}\right)^{4}\left(\frac{I_{2}^{\prime}-K_{2}^{\prime}+\nu_{2} E_{0}^{\prime}-6 \nu_{2} G_{0}^{\prime}}{I_{0}^{\prime}}\right)=0 .
\end{aligned}
$$

In other words, the plane (37) should lie in a particular four-dimensional space

$$
X_{2}-\nu_{2} X_{5}=0 \text {, }
$$

where $\nu_{2}$ is the solution of the above linear equation. To select $P_{5}$ in this space makes $\nu_{2}$ vanish, that is

$$
\frac{I_{0}^{\prime}-K_{0}^{\prime}}{I_{0}^{\prime}}\left(\frac{I_{2}}{I_{0}}+4 \frac{I_{2}^{\prime}}{I_{0}^{\prime}}\right)-5 \frac{I_{2}^{\prime}-K_{2}^{\prime}}{I_{0}^{\prime}}=0 .
$$

Since

$$
\begin{gathered}
I_{0}^{\prime}=6 b_{2,2} b_{3,3}, \quad K_{0}^{\prime}=4 b_{2,2} b_{3,3}, \\
I_{2}=0, \quad I_{2}^{\prime}=18 b_{2,2} b_{3,5}, \quad K_{2}^{\prime}=12 b_{2,2}^{\prime} b_{3,5},
\end{gathered}
$$

$\left(41^{\prime}\right)$ is equivalent to

$$
b_{3,5}=0 .
$$

If we further impose the condition that the covariant points $O_{8, j}(j=1,2,3)$ [6] also coincide

$$
\begin{aligned}
\frac{a_{0}^{5}}{b_{0}} U_{10,3}-A_{5,3}= & \left(\frac{I_{0}^{\prime}-K_{0}^{\prime}}{I_{0}^{\prime}}\right)^{5}\left(\frac{I_{3}}{I_{0}}+4 \frac{I_{3}^{\prime}-\vartheta J_{0}^{\prime}}{I_{0}^{\prime}}\right) \\
& -5\left(\frac{I_{0}^{\prime}-K_{0}^{\prime}}{I_{0}^{\prime}}\right)^{4}\left(\frac{I_{3}^{\prime}-K_{3}^{\prime}+\nu_{1} L_{0}^{\prime}-\nu_{1} J_{0}^{\prime}}{I_{0}}\right)=0 .
\end{aligned}
$$

The singular point $P$ of $\Gamma_{3}$ is then representable only when (40) and (43) are consistent. If we select $P_{5}$ in the common part of $x_{2}=0$ and $x_{3}-\nu_{2} x_{5}=0$, then $\nu_{1}$ vanishes,

$$
\left(I_{0}^{\prime}-K_{0}^{\prime}\right)\left(\frac{I_{3}}{I_{0}}+4 \frac{I_{3}^{\prime}}{I_{0}^{\prime}}\right)-5\left(I_{3}^{\prime}-K_{3}^{\prime}\right)=0 .
$$

From (8), (20), (22), (30), (33) and (40) we find that

$$
\begin{gathered}
I_{3}^{\prime}=-30 b_{2,5} b_{3,3}+48 b_{2,2} b_{3,6}, \quad I_{3}=30 b_{2,2} b_{3,3} b_{5,5}\left(\frac{b_{5,8}}{b_{5,5}}-\frac{2}{5} \frac{b_{3,6}}{b_{3,3}}\right), \\
K_{3}^{\prime}=28 b_{2,2} b_{3,6}-14 b_{2,5} b_{3,3} .
\end{gathered}
$$


(44) is now simplified to the form

$$
b_{5,8} / b_{5,5}+4 b_{2,5} / b_{2,2}-4 b_{3,6} / b_{3,3}=0 \text {. }
$$

In virtue of (36), $\left(36^{\prime}\right)$ and (46) it can also be written as

$$
b_{2,5} / b_{2,2}=11 \omega_{1} \text {. }
$$

The coordinates of the osculant $O_{10}$ are

$$
\begin{aligned}
& X_{2}=0, \quad X_{3}=0, \quad X_{4}=\nu_{3}, \\
& X_{1}=-\frac{I_{0}^{\prime}}{5 I_{0}}\left[\frac{1}{3}\left(\frac{I_{4}}{I_{0}}+4 \frac{I_{4}^{\prime}}{I_{0}^{\prime}}-\frac{4 J_{0}}{\rho I_{0}^{\prime}}\right)-5 \frac{I_{4}^{\prime}-K_{4}^{\prime}}{I_{0}^{\prime}}\right], \\
& X_{0}=\frac{I_{0}^{\prime}}{4 I_{0}}\left[\frac{I_{5}}{J_{0}}+4\left(\frac{I_{5}^{\prime}}{I_{0}^{\prime}}+\frac{\nu_{3} I_{0}}{\rho I_{0}^{\prime}}-\frac{J_{1}}{\rho I_{0}^{\prime}}\right)-15 \frac{I_{5}^{\prime}-K_{5}^{\prime}}{I_{0}^{\prime}}\right],
\end{aligned}
$$

whose locus is a quadric $Q_{5}$ :

$$
X_{2}=0, \quad X_{3}=0 \text {, }
$$

$$
\begin{aligned}
\frac{X_{0}}{X_{5}}= & \frac{I_{0}^{\prime}}{4 I_{0}}\left\{\frac{I_{5}}{I_{0}}+\frac{4 I_{5}^{\prime}}{I_{0}^{\prime}}-15 \frac{I_{5}^{\prime}-K_{5}^{\prime}}{I_{0}^{\prime}}\right\} \\
& +\frac{5}{4}\left(\frac{I_{0} X_{4}}{J_{0} X_{5}}-J_{1}\right)\left\{\frac{X_{1}}{X_{5}}+\frac{I_{0}^{\prime}}{5 I_{0}}\left[\frac{1}{3}\left(\frac{I_{4}}{I_{0}}+4 \frac{I_{4}^{\prime}}{I_{0}^{\prime}}\right)-4 \frac{I_{4}^{\prime}-K_{4}^{\prime}}{I_{0}^{\prime}}\right]\right\} .
\end{aligned}
$$

Since $P_{1}$ and $P_{2}$ lie on the quadric $Q_{5}$, there is a point $T(\neq P)$, through which the two generators of $Q_{5}$ pass through $P_{1}$ and $P_{4}$ respectively. We select $T$ as $P_{5}$. The equation of the quadric (49) now reduces to

$$
X_{2}=0, \quad X_{3}=0, \quad X_{1} X_{4}=\frac{4}{45} \cdot \frac{b_{4,4}}{b_{5,5}} X_{0} X_{5} .
$$

Thus we have

$$
\begin{gathered}
J_{1}=0, \quad \frac{1}{3}\left[\frac{I_{4}}{I_{0}}+4 \frac{I_{4}^{\prime}}{I_{0}^{\prime}}\right]-5 \frac{I_{4}^{\prime}-K_{4}^{\prime}}{I_{0}^{\prime}}=0, \\
\frac{I_{5}}{I_{0}}+\frac{4 I_{5}^{\prime}}{I_{0}^{\prime}}-15 \frac{I_{5}^{\prime}-K_{5}^{\prime}}{I_{0}^{\prime}}=0 .
\end{gathered}
$$

Calculating $J_{1}, I_{r}, I_{r}^{\prime}, K_{r}^{\prime}(r=4,5)$ as well as $I_{3}, I_{3}^{\prime}$ and $K_{3}^{\prime}$, we obtain

$$
\begin{aligned}
J_{1} & =6 b_{2,2} b_{3,3} b_{4,5}, \quad I_{4}=6 b_{2,2} b_{3,3} b_{5,5}\left(\frac{4}{3} \frac{b_{5,9}}{b_{5,5}}+\frac{b_{2,6}}{b_{2,2}}-5 \frac{b_{3,7}}{b_{3,3}}\right) \\
I_{4}^{\prime} & =-54 b_{2,6} b_{3,3}+70 b_{2,2} b_{3,7}, \quad K_{4}^{\prime}=-24 b_{3,3} b_{2,6}+40 b_{2,2} b_{3,7} \\
I_{5} & =56 b_{2,2} b_{3,3} b_{5,10}+16 b_{5,5} b_{2,7} b_{3,5}-54 b_{2,2} b_{5,5} b_{3,8} \\
I_{6}^{\prime} & =-84 b_{3,3} b_{2,7}+98 b_{2,2} b_{3,8}, \quad K_{5}^{\prime}=54 b_{2,2} b_{3,8}-36 b_{3,3} b_{2,7}
\end{aligned}
$$


Consequently, (51) becomes

$$
\begin{gathered}
b_{4,5}=0 \\
7 b_{5,9} / b_{5,5}-100 b_{8,7} / 3 b_{8,3}+40 b_{2,6} / b_{2,2}=0
\end{gathered}
$$

and

$$
28 b_{5,10} / 3 b_{5,5}+200 b_{2,7} / 3 b_{2,2}-50 b_{3,8} / b_{3,3}=0 .
$$

The pyramid $\left\{P, P_{1}, P_{2}, P_{3}, P_{4}, P_{6}\right\}$ thus obtained is called the fundamental pyramid of the curve at the point $P$.

4. Canonical expansions and projective Frenet-Serret formulae. There are five species of ordinary curves $\Gamma$ in a projective five-dimensional space. Every point of $\Gamma$ may be either an ordinary point of the associated curve $C$ or a $k$-ic $(k=6,7,8)$ point. The pyramid in the last section is only valid where every point of $\Gamma$ is an ordinary point of $C$, because in other cases the determination of $P_{2}$ requires a particular modification.

4.1. The case where every point of $\Gamma$ is also an ordinary point of the curve $C$. Usually, the osculating conic of $\Gamma$ has at $P$ with $C$ a contact of order equal to or less than four, that is

$$
\left(2 D_{0} B_{0} / C_{0}\right) C_{3}-B_{0} D_{3}-B_{3} D_{0} \neq 0 .
$$

Through a calculation of Wronskians we reach

and

$$
\begin{aligned}
& B_{3}=60 \prod_{3}^{5} b_{i, i}\left[4 \frac{b_{3,6}}{b_{3,3}}-28 \frac{b_{4,7}}{b_{4,4}}+32 \frac{b_{5,8}}{b_{5,5}}\right], \\
& C_{3}=\prod_{3}^{5} b_{i, i}\left[120 \frac{b_{3,6}}{b_{3,3}}-768 \frac{b_{4,7}}{b_{4,4}}+840 \frac{b_{5,8}}{b_{5,5}}\right], \\
& D_{3}=\prod_{2}^{5} b_{i, i}\left[48 \frac{b_{3,6}}{b_{3,3}}-240 \frac{b_{4,7}}{b_{4,4}}+240 \frac{b_{5,8}}{b_{5,5}}\right],
\end{aligned}
$$

$$
\begin{aligned}
& B_{4}=\prod_{3}^{5} b_{i, i}\left[840 \frac{b_{3,7}}{b_{3,3}}-3600 \frac{b_{4,8}}{b_{4,4}}+3240 \frac{b_{5,9}}{b_{5,5}}\right], \\
& C_{4}=\prod_{8}^{5} b_{i, i}\left[432 \frac{b_{3,7}}{b_{3,3}}-1680 \frac{b_{4,8}}{b_{4,4}}+1440 \frac{b_{5,9}}{b_{5,8}}\right], \\
& D_{4}=\prod_{2}^{5} b_{i, i}\left[-12 \frac{b_{2,8}}{b_{2,2}}+180 \frac{b_{3,7}}{b_{3,3}}-540 \frac{b_{4,8}}{b_{4,4}}+420 \frac{b_{5,9}}{b_{5,8}}\right] .
\end{aligned}
$$

Substituting (36) and $\left(36^{\prime}\right)$ in $(57)$ we have

$$
B_{3}=960 \cdot 83 \prod_{3}^{5} b_{i, i} \omega_{1}, \quad C_{3}=256 \cdot 129 \prod_{3}^{5} b_{i, i} \omega_{1}, \quad D_{3}=33 \cdot 256 \prod_{3}^{5} b_{i, i} \omega_{1} .
$$

Hence $\omega_{1} \neq 0$, because of $(56)$. 
We have selected the fundamental pyramid in $\S 3$, for which $P P_{2}$ is the projective normal of $C$, so that, as $C_{1}=0, D_{2}=0$,

$$
2\left(D_{0} B_{0} / C_{0}\right) C_{4}-D_{0} B_{4}-B_{0} D_{4}=0 \text {. }
$$

From (7) and (59) we obtain

$$
-4 b_{3,7} / b_{3,3}+5 b_{4,8} / b_{4,4}-2 b_{5,9} / b_{5,5}+b_{2,6} / b_{2,2}=0 .
$$

Putting

$$
b_{2,6} / b_{2,2}=244 \omega_{2}
$$

and using the relations (35), (54) and (60), we find

$$
b_{3,7} / b_{3,3}=591 \omega_{2}, \quad b_{4,8} / b_{4,4}=992 \omega_{2}, \quad b_{5,9} / b_{5,5}=1420 \omega_{2} .
$$

The expansions of the nonhomogeneous coordinates of $\Gamma$ must be

$$
\begin{aligned}
& x_{2}=b_{2,2}\left[x_{1}^{2}+11 \omega_{1} x_{1}^{5}+244 \omega_{2} x_{1}^{6}\right]+b_{2,7} x_{1}^{7}+(8), \\
& x_{3}=b_{3,3}\left[x_{1}^{3}+36 \omega_{1} x_{1}^{6}+591 \omega_{2} x_{1}^{7}\right]+b_{3,8} x_{1}^{8}+(9), \\
& x_{4}=b_{4,4}\left[x_{1}^{4}+72 \omega_{1} x_{1}^{7}+992 \omega_{2} x_{1}^{8}\right]+b_{4,9} x_{1}^{9}+(10), \\
& x_{5}=b_{5,5}\left[x_{1}^{5}+100 \omega_{1} x_{1}^{8}+1420 \omega_{2} x_{1}^{9}\right]+b_{5,10} x_{1}^{10}+(11) .
\end{aligned}
$$

The unit point in the plane $\left\{P P_{1} P_{2}\right\}$ can be chosen in the same way as in \$1.3. Therefore the cubic (10) is to be given by the equation $-X_{0}^{2} X_{2}+X_{1}^{2} X_{0} / 2$ $+X_{1} X_{2}^{2} / 2=0$. Hence $D_{0} B_{0}=C_{0}^{2} / 2,-2 B_{0}^{2} C_{3} / C_{0}^{2} D_{0}+B_{0}^{2} D_{3} / C_{0} D_{0}^{2}+B_{0} B_{3} / C_{0} D_{0}$ $=1 / 2$, whence,

$$
b_{2,2}=4 / 5, \quad \omega_{1}=-1 / 500 .
$$

We further select the unit point in space such that $(1,1,1,1,0,0)$, $(1,1,0,1,1,0),(1,1,0,0,1,1)$ lie on $Q_{3}, Q_{4}$ and $Q_{5}$ respectively. This implies

$$
b_{3,3} / b_{2,2}=6 / 5, \quad b_{4,4} / b_{3,3}=3 / 10, \quad b_{5,5} / b_{4,4}=4 / 45 .
$$

The expansions (63) now are of the form

$$
\begin{aligned}
& x_{2}=\frac{4}{5}\left(x_{1}^{2}-\frac{11}{4 \cdot 5^{3}} x_{1}^{5}+244 \omega_{2} x_{1}^{6}\right)+b_{2,7} x_{1}^{7}+(8), \\
& x_{3}=\frac{24}{25}\left(x_{1}^{8}-\frac{36}{4 \cdot 5^{3}} x_{1}^{6}+591 \omega_{2} x_{1}^{7}\right)+b_{3,8} x_{1}^{8}+(9), \\
& x_{4}=\frac{36}{125}\left(x_{1}^{4}-\frac{72}{4 \cdot 5^{3}} x_{1}^{7}+992 \omega_{2} x_{1}^{8}\right)+b_{4,9} x_{1}^{9}+(10), \\
& x_{5}=\left(\frac{2}{5}\right)^{4}\left(x_{1}^{5}-\frac{1}{5} x_{1}^{8}+1420 \omega_{2} x_{1}^{9}\right)+b_{5,10} x_{1}^{10}+(11)
\end{aligned}
$$


Let $\left(P_{l}\right)(l=1, \cdots, 5)$ be the locus of $P_{l}$, and let $z_{l}^{j}(u)(j=1, \cdots, 6)$ and $z^{j}(u)$ be the homogeneous coordinates of the curves $\left(P_{\downarrow}\right)(l=1, \cdots, 5)$ and $\Gamma$ with respect to a certain coordinate system; then from the definition of the fundamental pyramid there exist functions $a, b$ and $a_{l m}$ such that

(67') $\frac{d z}{d u}=a z+b z_{1}, \frac{d z_{s}}{d u}=\sum_{j=0}^{s+1} a_{s, j} z_{j}, \frac{d z_{5}}{d u}=\sum_{j=0}^{s} a_{6, j} z_{j} \quad(s=1,2,3,4)$.

Evidently there can be determined, save for a constant factor, a unique function $\sigma(u)$ such that

$$
d(\sigma z) / d u=a z_{1} .
$$

Hereafter the coordinates $z^{j}(u)$ and $z_{l}^{j}(u)(l=1, \cdots, 5 ; j=1, \cdots, 6)$ multiplied by $\sigma(u)$ are still denoted by $z^{j}(u), z_{l}^{j}(u)$. The equations $\left(67^{\prime}\right)$ now reduce to

$$
\frac{d z}{d u}=a z_{1}, \quad \frac{d z_{s}}{d u}=\sum_{j=0}^{s+1} a_{s, j} z_{j}, \quad \frac{d z_{5}}{d u}=\sum_{j=0}^{5} a_{5, j} z_{j} \quad(s=1,2,3,4) .
$$

If $x_{1}, \cdots, x_{5}$ are the nonhomogeneous coordinates of a fixed point in the space with respect to the local coordinate system thus determined, the condition of immovability is

$$
\frac{d}{d u}\left(z+\sum_{j=1}^{5} x_{j} z_{j}\right)=\lambda(u)\left(z+\sum_{j=1}^{5} x_{j} z_{j}\right)
$$

or

$$
\begin{aligned}
\lambda(u)=\sum_{i=1}^{5} a_{i, 0} x_{i}, & \lambda x_{1}=a+\frac{d x_{1}}{d u}+\sum_{i=1}^{5} a_{i, 1} x_{i}, \\
\lambda x_{k}=\frac{d x_{k}}{d u}+\sum_{i=1}^{5} a_{i, k} x_{i} & (k=2,3,4,5) .
\end{aligned}
$$

Therefore the elimination of $\lambda, d x_{1} / d u$ and $x_{k}(k=2, \cdots, 5)$ from (66) and (68) gives four equations. Consequently, we have the relations :

$$
\left\{\begin{array}{c}
a_{3,4}=6 a / 5, \quad a_{4,4}=4 a_{1,1}, \quad 3 a_{1,0}-16 a_{2,1} / 5+4 a_{5,4} / 45=0 \\
\cdot a_{2,0}-8 a_{3,1} / 5+21 a / 50-a_{3,4} / 10=0 \\
72 a_{3,0} / 25-8 \cdot 992 a \omega_{2}+126 a_{1,1} / 625-144 a_{4,1} / 625 \\
+1970 a_{3,4} \omega_{2}-2 a_{4,4} / 5=0 \\
36 \cdot 548 a_{2,1} / 5^{7}-18 \cdot(2 / 5)^{7} a_{5,1}+3^{5} \cdot 2^{4} a_{4,0} / 5^{6}-3^{5} \cdot 2^{4} a_{1,0} / 5^{6} \\
-2^{10} \cdot 9 \cdot 31 a_{1,1} \omega_{2} / 5^{3}-2^{4} a_{5,4} / 5^{5}-9 a b_{4,9} \\
+\left(2^{7} \cdot 279 / 5^{3}\right) d \omega_{2} / d u+a_{3,4} b_{3,8}+279 \cdot 2^{7} a_{4,4} \omega_{2} / 625=0
\end{array}\right.
$$




$$
\begin{gathered}
a_{4,5}=4 a / 9, \quad a_{5,5}=5 a_{1,1}, \quad a_{1,0}=a_{2,1}, \\
2^{4} a_{2,0}-24 a_{3,1}+8 a-81 a_{4,5} / 10=0,
\end{gathered}
$$

$\left(69_{2}\right) \quad 96 a_{3,0} / 25-36 a_{4,1} / 25+8 a_{1,1} / 5-11160 a \omega_{2}+248 \cdot 45 a_{4,5} \omega_{2}-a_{5,5} / 5=0$, $4^{4} \cdot 9 a_{4,0} / 5^{7}-7 \cdot 2^{4} a_{1,0} / 5^{5}+11 \cdot 2^{4} a_{2,1} / 5^{7}-2^{8} a_{5,1} / 5^{7}+2^{9} a_{2,1} / 5^{6}$

$$
-10 a b_{5,10}+\left(71 \cdot 2^{6} / 5^{3}\right) d \omega_{2} / d u+a_{4,5} b_{4,9}-2^{6} \cdot 284 a_{1,1} \omega_{2} / 5^{3}=0 ;
$$

$$
a_{1,2}=8 a / 5, \quad a_{2,2}=2 a_{1,1}, \quad a_{1,0}=2 a_{3,2},
$$

$$
80 a_{2,0}-192 a_{3,1}+11 a+36 a_{4,2}=0,
$$

$15 \cdot 2^{5} a_{3,0}-9 \cdot 2^{5} a_{4,1}+55 a_{1,1}-732000 a \omega_{2}-11 a_{2,2}+16 a_{5,2}=0$,

$144 a_{4,0} / 5^{4}+a_{1,0}\left(-88 / 5^{4}\right)+28 \cdot 11 a_{2,1} / 5^{5}-2^{7} a_{5,1} / 5^{5}$

$-183 \cdot 2^{5} a_{1,1} \omega_{2} / 5-7 b_{2,7} a+(976 / 5) d \omega_{2} / d u$

$$
+976 a_{2,2} \omega_{2} / 5-6^{3} a_{3,2} / 5^{5}=0 \text {; }
$$

$$
\left\{\begin{array}{c}
a_{2,3}=18 a / 5, \quad a_{3,3}=3 a_{1,1}, \quad 20 a_{1,0}-24 a_{2,1}+3 a_{4,3}=0 \\
2^{6} \cdot 15 a_{2,0}-3^{3} \cdot 2^{6} a_{3,1}+6^{4} a / 5-11 a_{2,3}+16 a_{5,3}=0, \\
1152 a_{3,0}-3^{3} \cdot 2^{6} a_{3,1} / 5+6^{4} a / 5-11 a_{2,3}+16 a_{5,3}=0 \\
3^{8} \cdot 2^{6} a_{4,0} / 5^{5}-6^{3} a_{1,0} / 5^{4}+(216 \cdot 197 / 25) d \omega_{2} / d u-8 a b_{3,8} \\
-197 \cdot 288 a_{1,1} \omega_{2} / 25+a_{2,1}\left(1656 / 5^{6}\right)-1152 a_{5,1} / 5^{6} \\
+a_{2,3} b_{2,7}-3^{4} \cdot 8 a_{4,3} / 5^{6}=0 .
\end{array}\right.
$$

Some simple calculations show that $\left(69_{i}\right)(i=1,2,3,4)$ are equivalent to

$$
\begin{aligned}
& a_{1,2}=8 a / 5, \quad a_{2,3}=18 a / 5, \quad a_{3,4}=6 a / 5, \quad a_{4,5}=4 a / 9, \\
& a_{2,2}=2 a_{1,1}, \quad a_{3,3}=3 a_{1,1}, \quad a_{4,4}=4 a_{1,1}, \quad a_{5,5}=5 a_{1,1}, \\
& \text { (69') } a_{2,1}=a_{1,0}, \quad a_{3,2}=a_{1,0} / 2, \quad a_{4,3}=4 a_{1,0} / 3, \quad a_{5,4}=9 a_{1^{\prime}, 0} / 4 \text {, } \\
& a_{2,0}=a / 10, \quad a_{3,1}=a / 4, \quad a_{5,3}=291 a / 40, \quad a_{4,2}=29 a / 36 \text {, }
\end{aligned}
$$

and

$$
\begin{aligned}
7 a b_{2,7}= & 144 a_{4,0} / 5^{4}-4 a_{1,0} / 5^{4}-784 a_{1,1} \omega_{2}+\left(2^{4} \cdot 61 / 5\right) d \omega_{2} / d u-2^{7} a_{5,1} / 5^{5}, \\
8 a b_{3,8}= & 17 \cdot 3^{3} \cdot 2^{5} a_{4,0} / 7 \cdot 5^{5}+88 \cdot 27 a_{1,0} / 5^{6} \cdot 7+\left(3^{3} \cdot 8 \cdot 541 / 27 \cdot 5\right) d \omega_{2} / d u \\
& -232 \cdot 288 a_{1,1} \omega_{2} / 25-3^{4} \cdot 2^{7} a_{5,1} / 5^{6} \cdot 7, \\
\left(69^{\prime \prime}\right) \quad 9 a b_{4,9}= & -9 \cdot 863 a_{1,0} / 14 \cdot 5^{7}+3^{4} \cdot 472 a_{4,0} / 5^{6} \cdot 7-2^{5} \cdot 747 a_{5,1} / 7 \cdot 5^{7} \\
& -603 \cdot 2^{6} a_{1,1} \omega_{2} / 25+\left(18 \cdot 18757 / 5^{3} \cdot 7\right) d \omega_{2} / d u, \\
10 a b_{5,10}= & 2^{5} \cdot 799 a_{4,0} / 5^{7} \cdot 7-5758 a_{1,0} / 5^{7} \cdot 63-2^{7} \cdot 209 a_{5,1} / 5^{7} \cdot 63
\end{aligned}
$$$$
-2^{6} \cdot 3299 a_{1,1} \omega_{2} / 125+\left(8 \cdot 54541 / 5^{3} \cdot 63\right) d \omega_{2} / d u \text {. }
$$ 
By use of (55)

$$
\begin{aligned}
a_{4,0}= & 50978 a_{1,0} / 997 \cdot 252-9256 a_{5,1} / 6993+5^{4} \cdot 160544 a_{1,1} \omega_{2} / 997 \\
& +\left(5^{4} \cdot 1334107 / 36 \cdot 997\right) d \omega_{2} / d u .
\end{aligned}
$$

Thus (67) becomes

$$
\begin{aligned}
d z / d u= & a z_{1}, \\
d z_{1} / d u= & a\left(I_{1} z-5^{3} \cdot 3^{3} I_{2} z_{1} / 2+8 z_{2} / 5\right), \\
d z_{2} / d u= & a\left(z / 10+I_{1} z_{1}-5^{3} \cdot 3^{3} I_{2} z_{2}+18 z_{3} / 5\right), \\
d z_{3} / d u= & a\left(I_{2}\left(5^{4} \cdot 51 z / 8\right)+z_{1} / 4+I_{1} z_{2} / 2-5^{3} \cdot 3^{4} I_{2} z_{3} / 2+6 z_{4} / 5\right), \\
d z_{4} / d u= & a\left[( 1 / 9 9 7 ) \left(50987 I_{1} / 252-9256 I_{3} / 9-5^{7} \cdot 3^{3} \cdot 80272 I_{2}^{2}\right.\right. \\
& \left.+\left(5^{4} \cdot 1334107 / 36\right) d I_{2} / a d u\right) z+2587 \cdot 5^{3} I_{2} z_{1} / 72+29 z_{2} / 36 \\
& \left.+4 I_{1} z_{3} / 3-250 \cdot 3^{3} I_{2} z_{4}+4 z_{5} / 9\right], \\
d z_{5} / d u= & a\left[I_{4} z+I_{3} z_{1}+5^{3} \cdot 2099 I_{2} z_{2} / 2^{5}+291 z_{3} / 40\right. \\
& \left.+9 I_{1} z_{4} / 4-5^{4} \cdot 3^{3} I_{2} z_{5} / 2\right],
\end{aligned}
$$

where we have denoted $a_{1,0}, a_{5,1}, a_{5,0}$ and $\omega_{2}$ by $a I_{1}, a I_{3}, a I_{4}$ and $I_{2}$ respectively. Evidently, $a, I_{i}(i=1,2,3,4)$ are five projective invariants while $a d u$ is an intrinsic form. Putting $a d u=d \sigma$ we have

$$
\begin{aligned}
d z / d \sigma= & z_{1}, \\
d z_{1} / d \sigma= & I_{1} z-5^{3} \cdot 3^{3} I_{2} z_{1} / 2+8 z_{2} / 5 \\
d z_{2} / d \sigma= & z / 10+I_{1} z_{1}+(-15)^{3} I_{2} z_{2}+18 z_{3} / 5 \\
d z_{3} / d \sigma= & 5^{4} \cdot 51 I_{2} z / 8+z_{1} / 4+I_{1} z_{2} / 2-5^{3} \cdot 3^{4} I_{2} z_{3} / 2+6 z_{4} / 5 \\
\text { (A) } d z_{4} / d \sigma= & (1 / 997)\left(50987 I_{1} / 252-9256 I_{3} / 9+\left(5^{4} \cdot 1334107 / 36\right) d I_{2} / d \sigma\right. \\
& \left.-5^{7} \cdot 3^{3} \cdot 80272 I_{2}^{2}\right) z+5^{3} \cdot 2587 I_{2} z_{1} / 72+29 z_{2} / 36 \\
& +4 I_{1} z_{3} / 3-3^{3} \cdot 250 I_{2} z_{4}+4 z_{5} / 9 \\
d z_{5} / d \sigma= & I_{4} z+I_{3} z_{1}+5^{3} \cdot 2099 I_{2} z_{2} / 2^{5}+291 z_{3} / 40 \\
& +9 I_{1} z_{4} / 4-5^{4} \cdot 3^{3} I_{2} z_{5} / 2 .
\end{aligned}
$$

Either $\left(\epsilon_{1}^{2}, \epsilon_{1}, 1\right)$ or $\left(\epsilon_{2}^{2}, \epsilon_{2}, 1\right)$ can be taken for the unit point in the plane $\left\{P P_{1} P_{2}\right\}$ (cf. $\left.\S \S 1.3,1.4\right)$; similarly, each of the two points $\left(\epsilon_{j}^{2}, \epsilon_{j}, 1, \epsilon_{j}^{2}, \epsilon_{j}, 1\right)$, $\left(\epsilon_{j}^{3}=1, \epsilon_{j} \neq 1, j=1,2\right)$ can be taken for the unit point in the five-dimensional space. This gives rise to a projective transformation $T$, under which $z, z_{1}, z_{2}$, $z_{3}, z_{4}, z_{5}$ correspond to $\epsilon_{j}^{2} z, \epsilon_{j} z_{1}, z_{2}, \epsilon_{j}^{2} z_{3}, \epsilon_{j} z_{4}, z_{5}$ respectively. Accordingly $d \sigma, I_{1}, I_{2}$ and $I_{3}$ are transformed to $\epsilon_{j} d \sigma, \epsilon_{j} I_{1}, \epsilon_{j}^{2} I_{2}, \epsilon_{j} I_{3}$ but $I_{4}$ is invariant.

Furthermore, elimination of $z_{1}, z_{2}, z_{3}, z_{4}$ and $z_{5}$ from the equations (A) gives a differential equation of order six (B) invariant with respect to $T$. The curve in consideration now possesses four invariants $I_{i}(i=1, \cdots, 4)$ and 
an arc-element $d \sigma$. Given four analytic functions $I_{j}(\sigma)(j=1, \cdots, 4)$, the coordinates of a curve in five-dimensional space having $I_{j}(\sigma)$ as its projective invariants and $d \sigma$ as its projective arc-element are independent solutions of the differential equation (B).

4.2. The Frenet-Serret formulae in the case where $C$ always has $P$ for its sextactic point. Since no projective normal can be drawn at a sextactic point of a plane curve, the vertex $P_{2}$ in the present case cannot be determined as before. But the way of determining the canonical expansion of a plane curve at its sextactic point furnishes a covariant triangle $\left\{P P_{1} P_{2}\right\}$ and a unit point in the osculating plane so that the other vertices of the covariant pyramid and the covariant unit point in the five-dimensional space can also be defined without any difficulty.

In fact, from (7) and $2\left(D_{0} B_{0} / C_{0}\right) C_{3}-B_{0} D_{3}-B_{3} D_{0}=0$, we have

$$
\omega_{1}=0 \text {. }
$$

Hence neither (59) nor (60) exists.

From the definition of the triangle $\left\{P P_{1} P_{2}\right\}$ and (21) and (23) in $\$ 2.1$ it follows that the quartic

$$
\begin{aligned}
& 8 X_{2}^{3}\left(X_{2}-2 X_{1}\right)-8 X_{2}^{3}\left(2 X_{1}-2 X_{0}-X_{2} / 2\right) \\
& +\left(2 X_{1}-2 X_{0}-X_{2} / 2\right)^{2}\left(4 X_{1}^{2}-4 X_{0} X_{2}\right)=0
\end{aligned}
$$

has at $P$ a contact of order seven with $C$. Since $B_{i}=0, C_{i}=0, D_{i}=0$ $(i=1,2,3)$, we obtain after substituting $(4)$ in $(72)$ that

$$
\begin{gathered}
C_{0}^{2}-B_{0} D_{6}=0, \quad D_{0}^{3}+B_{0}\left(2 C_{0} C_{4}-B_{0} D_{4}-B_{4} D_{0}\right)=0, \\
2 C_{0} C_{5}-B_{0} D_{5}-B_{5} D_{0}=0,
\end{gathered}
$$

which are equivalent to

$$
\begin{gathered}
b_{2,2}=8 / 5 \\
(2 / 5)^{4}+b_{2,6} / b_{2,2}-4 b_{3,7} / b_{3,3}+5 b_{4,8} / b_{4,4}-2 b_{5,9} / b_{5,5}=0 \\
8 b_{2,7} / b_{2,2}-20 b_{3,8} / b_{3,3}+20 b_{4,9} / b_{4,4}-7 b_{5,10} / b_{5,5}=0
\end{gathered}
$$

because of (7) and

$$
\begin{aligned}
& B_{5}=60 \prod_{8}^{5} b_{i, i}\left[2^{5} \cdot \frac{b_{3,8}}{b_{3,3}}-3^{3} \cdot 4 \frac{b_{4,9}}{b_{4,4}}+84 \frac{b_{5,10}}{b_{5,5}}\right], \\
& C_{5}=\prod_{3}^{5} b_{i, i}\left[7 \cdot 144 \frac{b_{3,8}}{b_{3,3}}-6 \cdot 8^{3} \frac{b_{4,9}}{b_{4,4}}+28 \cdot 3^{4} \frac{b_{5,10}}{b_{5,5}}\right], \\
& D_{5}=48 \prod_{2}^{5} b_{i, i}\left[-\frac{b_{2,7}}{b_{2,2}}+9 \frac{b_{3,8}}{b_{3,3}}-21 \frac{b_{4,9}}{b_{4,4}}+14 \frac{b_{5,10}}{b_{5,5}}\right] .
\end{aligned}
$$


Combining (65) and (74) we obtain

$$
b_{3,8}=48 / 25, \quad b_{4,4}=72 / 125, \quad b_{5,5}=2^{5} / 5^{4} .
$$

For convenience we put

$$
b_{5,9} / b_{5,5}=\omega_{2}
$$

then (35), (54), (75) and (77) give

$$
\begin{aligned}
& b_{2,6} / b_{2,2}=61 \omega_{2} / 355+(45 / 71) \cdot(2 / 5)^{4} \\
& b_{3,7} / b_{3,3}=591 \omega_{2} / 1420+(54 / 71) \cdot(2 / 5)^{4} \\
& b_{4,8} / b_{4,4}=248 \omega_{2} / 355+(20 / 71) \cdot(2 / 5)^{4}
\end{aligned}
$$

The expansions of the coordinates of $\Gamma$ are no longer(66), but take the form

$$
\begin{aligned}
& x_{2}=\frac{8}{5}\left[x_{1}^{2}+\left(\frac{61 \omega_{2}}{355}+\frac{45}{71} \cdot\left(\frac{2}{5}\right)^{4}\right) x_{1}^{6}\right]+b_{2,7} x_{1}^{7}+(8), \\
& x_{3}=\frac{48}{25}\left[x_{1}^{3}+\left(\frac{591 \omega_{2}}{1420}+\frac{54}{71} \cdot\left(\frac{2}{5}\right)^{4}\right) x_{1}^{7}\right]+b_{3,8} x_{1}^{8}+(9), \\
& x_{4}=\frac{72}{125}\left[x_{1}^{4}+\left(\frac{248 \omega_{2}}{355}+\frac{20}{71} \cdot\left(\frac{2}{5}\right)^{4}\right) x_{1}^{8}\right]+b_{4,9} x_{1}^{9}+(10), \\
& x_{5}=\frac{2^{5}}{5^{4}}\left[x_{1}^{5}+\left(\omega_{2}\right) x_{1}^{9}\right]+b_{5,10} x_{1}^{10}+(11) .
\end{aligned}
$$

However, there are, as in the former case, functions $a_{i j}(u)$ and $a(u)$ satisfying (67). Some computations suffice to demonstrate that

$$
\begin{aligned}
& a_{1,2}=16 a / 5, \quad a_{2,3}=18 a / 5, \quad a_{3,4}=6 a / 5, \quad a_{4,5}=4 a / 9, \\
& a_{2,2}=2 a_{1,1}, \quad a_{3,3}=3 a_{1,1}, \quad a_{4,4}=4 a_{1,1}, \quad a_{5,6}=5 a_{1,1}, \\
& a_{2,1}=a_{1,0} / 2, \quad a_{3,2}=a_{1,0} / 2, \quad a_{4,3}=4 a_{1,0} / 3, \quad a_{5,4}=9 a_{1,0} / 4 \text {, } \\
& a_{2,0}=0, \quad a_{3,1}=0, \quad a_{4,2}=0, \quad a_{5,3}=0,
\end{aligned}
$$

as well as eight other relations

$$
\begin{aligned}
& -\frac{72}{25} a_{4,1}-3 a\left(\frac{61 \omega_{2}}{71}+\frac{144}{71 \cdot 25}\right)+\frac{2}{25} \cdot a_{5,2}=0 \\
& -\frac{72}{25} a_{4,1}+\frac{32}{5} a_{3,0}-\frac{1135 a}{284} \omega_{2}-\frac{6^{4}}{71 \cdot 5^{3}} a=0, \\
& -\frac{72}{25} a_{4,1}+\frac{36}{5} a_{3,0}-\frac{1393 a}{284} \omega_{2}+\frac{2^{5 \cdot 7}}{71 \cdot 5^{3}} a=0 \\
& -\frac{24}{25} a_{4,1}+\frac{64}{25} a_{3,0}-\frac{391 a}{213} \omega_{2}+\frac{2^{6}}{213 \cdot 25} a=0,
\end{aligned}
$$




$$
\begin{aligned}
& \frac{b_{2,7}}{b_{2,2}}=\frac{1}{7 a}\left(a_{4,0} \cdot \frac{72}{125}-\frac{2^{6}}{5^{4}} a_{5,1}-4 a_{1,1} \frac{b_{2,6}}{b_{2,2}}\right), \\
& \frac{b_{3,8}}{b_{3,3}}=\frac{1}{8 a}\left[\frac{72 \cdot 31}{5^{3} \cdot 7} a_{4,0}-\frac{3^{3} \cdot 2^{5}}{7 \cdot 5^{4}} a_{5,1}+4 a_{1,1}\left(-\frac{b_{3,7}}{b_{3,3}}-\frac{3}{7} \frac{b_{2,6}}{b_{2,2}}\right)\right],
\end{aligned}
$$

$\left(81^{\prime \prime}\right) \frac{b_{4,9}}{b_{4,4}}=\frac{1}{9 a}\left[\frac{73 \cdot 36}{5^{3} \cdot 7} a_{4,0}-\frac{2^{4} \cdot 83}{5^{4} \cdot 7} a_{5,1}+2 a_{1,1}\left(-\frac{2 b_{4,8}}{b_{4,4}}-\frac{b_{3,7}}{b_{3,3}}-\frac{3}{7} \frac{b_{2,6}}{b_{2,2}}\right)\right]$,

$$
\begin{aligned}
\frac{b_{5,10}}{b_{5,5}}=\frac{1}{10 a} & {\left[\frac{-2^{5} \cdot 30187}{9^{2} \cdot 5^{4} \cdot 7} a_{4,0}-\frac{2^{5} \cdot 128239}{5^{5} \cdot 7 \cdot 3^{16}} a_{5,1}\right.} \\
+ & \left.\frac{2^{5} a_{1,1}}{5 \cdot 9^{3}}\left(-\frac{9^{3} \cdot 5}{8} \frac{b_{5,9}}{b_{5,5}}-\frac{2 b_{4,8}}{b_{4,4}}-\frac{b_{3,7}}{b_{3,3}}-\frac{3}{7} \frac{b_{2,6}}{b_{2,2}}\right)\right] .
\end{aligned}
$$

From $\left(81^{\prime}\right)$ we find

$$
\begin{aligned}
\omega_{2} & =2^{6} \cdot 5413 / 525 \cdot 4421, \\
a_{3,0} & =(6 a / 5) \cdot\left[2^{4} \cdot 19 / 71 \cdot 5^{2}-129 \omega_{2} / 142\right], \\
a_{4,1} & =a\left(1358 / 3195-25 \cdot 3199 \omega_{2} / 71 \cdot 9 \cdot 2^{5}\right), \\
a_{5,2} & =a\left(2^{4} \cdot 407 / 355+61675 \omega_{2} / 568\right) .
\end{aligned}
$$

Substituting $\left(81^{\prime \prime}\right)$ in (55) and (76) we are led to

$$
a_{4,0}=C_{1} a_{1,1}, \quad a_{5,1}=C_{2} a_{1,1},
$$

where $C_{1}$ and $C_{2}$ are two constants. If the invariant form $a d u$ be denoted by $d \sigma$, and the projective invariants $a_{1,0} / a, a_{1,1} / a$ and $a_{5,0} / a$ by $I_{1}, I_{2}$ and $I_{3}$ respectively, then the projective Frenet-Serret formulae read:

(C)

$$
\begin{aligned}
& \frac{d z}{d \sigma}=z_{1}, \quad \frac{d z_{1}}{d \sigma}=I_{1} z+I_{2} z_{1}+\frac{16}{5} z_{2}, \quad \frac{d z_{2}}{d \sigma}=\frac{1}{2} I_{1} z_{1}+2 I_{2} z_{2}+\frac{18}{5} z_{3}, \\
& \frac{d z_{3}}{d \sigma}=\frac{6}{355}\left(\frac{16 \cdot 19}{25}-\frac{129}{2} \omega_{2}\right) z+\frac{I_{1}}{2} z_{2}+3 I_{2} z_{3}+\frac{6}{5} z_{4},
\end{aligned}
$$

$$
\begin{aligned}
& \frac{d z_{4}}{d \sigma}=C_{1} I_{2} z+\frac{1}{639}\left(\frac{1358}{5}-\frac{5^{2} \cdot 3199}{2^{5}} \omega_{2}\right) z_{1}+\frac{4 I_{1}}{3} z_{3}+4 I_{2} z_{4}+\frac{4}{9} z_{5}, \\
& \frac{d z_{5}}{d \sigma}=I_{3} z+C_{2} I_{2} z_{1}+\frac{25}{71}\left(-\frac{2467}{8} \omega_{2}+\frac{2^{4} \cdot 407}{5^{3}}\right) z_{2}+\frac{9 I_{1}}{4} z_{4}+5 I_{2} z_{5} .
\end{aligned}
$$

In this case four points can be taken for the unit point without changing the invariants $I_{1}(\sigma), I_{2}(\sigma), I_{3}(\sigma)$ and the form $d \sigma$ save for a constant factor. The differential equation obtained from (C) by eliminating $z_{i}(i=1,2,3,4,5)$ is evidently independent of these various selections $\left({ }^{5}\right)$.

(5) We can reach similar results in $\$ \$ 4.3$ and 4.4 . 
4.3. The case where every point of $\Gamma$ is a 7-ic point of the curve $C$. When $P$ is a 7 -ic point, the osculating conic of $\Gamma$ at $P$ has at $P$ a contact of order six with $C$. Besides $\omega_{1}=0$, we have

$$
2\left(B_{0} D_{0} / C_{0}\right) C_{4}-B_{0} D_{4}-B_{4} D_{0}=0
$$

or its equivalent

$$
-4 b_{3,7} / b_{3,3}+5 b_{4,8} / b_{4,4}+b_{2,6} / b_{2,2}-2 b_{6,9} / b_{5,5}=0 .
$$

We now have to take the fundamental triangle of the plane curve $C$ at $P$ as the triangle $\left\{P P_{1} P_{2}\right\}$ and select the unit point in this plane by the method stated in $\$ 2.2$. This implies that the quartic $-2 X_{1} X_{2}^{3} / 3+X_{0}^{2}\left(X_{1}^{2}-X_{0} X_{2}\right)=0$ has at $P$ a contact of order eight with $C$. We then have the following conditions

$$
\begin{gathered}
C_{0}^{2}-B_{0} D_{0}=0, \quad-2 C_{0} D_{0}^{3} / 3+B_{0}^{2}\left(2 C_{0} C_{5}-B_{0} D_{5}-B_{6} D_{0}\right)=0, \\
2 C_{0} C_{6}-B_{0} D_{6}-B_{6} D_{0}=0,
\end{gathered}
$$

which, in turn, can be expressed in terms of $b_{i, j}$ as follows:

$$
b_{2,2}=8 / 5 \text {, }
$$

use being made of (7) and

$$
\begin{aligned}
& -\frac{2^{4}}{3 \cdot 5^{5}}+8 \frac{b_{2,7}}{b_{2,2}}-20 \frac{b_{3,8}}{b_{3,3}}+20 \frac{b_{4,9}}{b_{4,4}}-7 \frac{b_{5,10}}{b_{5,5}}=0, \\
& 12 \frac{b_{2,8}}{b_{2,2}}+5 \frac{b_{3,9}}{b_{3,3}}-21 \frac{b_{4,10}}{b_{4,4}}+28 \cdot\left(\frac{3}{5}\right)^{2} \frac{b_{5,11}}{b_{5,5}}=0
\end{aligned}
$$

$$
\begin{aligned}
& B_{5}=\prod_{3}^{5} b_{i, i}\left[15 \cdot 2^{7} \frac{b_{3,8}}{b_{3,3}}-80 \cdot 3^{4} \frac{b_{4,9}}{b_{4,4}}+5040 \frac{b_{5,10}}{b_{5,5}}\right], \\
& C_{5}=\prod_{3}^{5} b_{i, i}\left[1008 \frac{b_{3,8}}{b_{3,3}}-3 \cdot 2^{10} \frac{b_{4,9}}{b_{4,4}}+28 \cdot 3^{4} \frac{b_{5,10}}{b_{5,5}}\right], \\
& D_{5}=\prod_{2}^{5} b_{i, i}\left[-3 \cdot 2^{4} \frac{b_{2,7}}{b_{2,2}}+3^{3} \cdot 16 \frac{b_{3,8}}{b_{3,3}}-63 \cdot 16 \frac{b_{4,9}}{b_{4,4}}+21 \cdot 2^{5} \frac{b_{5,10}}{b_{5,5}}\right], \\
& B_{6}=\prod_{3}^{5} b_{i, i}\left[3600 \frac{b_{3,9}}{b_{3,3}}-10500 \frac{b_{4,10}}{b_{4,4}}+231 \cdot 2^{5} \frac{b_{5,11}}{b_{5,5}}\right], \\
& C_{6}=\prod_{3}^{5} b_{i, i}\left[1920 \frac{b_{3,9}}{b_{3,3}}-5040 \frac{b_{4,10}}{b_{4,4}}+210 \cdot 16 \frac{b_{5,11}}{b_{5,5}}\right], \\
& D_{6}=\prod_{2}^{5} b_{i, i}\left[-120 \frac{b_{2,8}}{b_{2,2}}+840 \frac{b_{3,9}}{b_{3,3}}-1680 \frac{b_{4,9}}{b_{4,4}}+63 \cdot 16 \frac{b_{5,11}}{b_{5,5}}\right] .
\end{aligned}
$$

Since $b_{2,2}$ has the same numerical value as in the former case, the equations 
(77) and (81) remain valid. From (35), (54) and (85) we find, by putting

that

$$
b_{5,9} / b_{5,5}=\omega_{2}
$$

(90) $\quad b_{2,8} / b_{2,2}=61 \omega_{2} / 71 \cdot 5, \quad b_{3,7} / b_{3,3}=591 \omega_{2} / 1420, \quad b_{4,8} / b_{4,4}=248 \omega_{2} / 355$.

$\left(81^{\prime}\right)$ must now be replaced by the four independent equations

$$
\begin{array}{r}
-72 a_{4,1} / 25-183 a \omega_{2} / 71+2 a_{5,2} / 25=0, \\
-72 a_{4,1} / 25+2^{5} a_{3,0} / 5-1135 a \omega_{2} / 284=0, \\
-72 a_{4,1} / 25+36 a_{3,0} / 5-1393 a \omega_{2} / 284=0, \\
-24 a_{4,1} / 25+64 a_{3,0} / 25-391 a \omega_{2} / 213=0,
\end{array}
$$

linear in $\omega_{2}, a_{4,1}, a_{5,2}$ and $a_{8,0}$. Thus

$$
\omega_{2}=0, \quad a_{4,1}=0, \quad a_{5,2}=0, \quad a_{3,0}=0 .
$$

Consequently $\left(81^{\prime \prime}\right)$ reduce to

(93)

$$
\begin{array}{r}
a_{4,0} \cdot \frac{72}{125}-\frac{64}{625} a_{5,1}-\frac{7 a b_{2,7}}{b_{2,2}}=0, \\
a_{4,0} \cdot \frac{144}{125}-\frac{96}{625} a_{5,1}+3 a \frac{b_{2,7}}{b_{2,2}}-8 a \frac{b_{3,8}}{b_{3,3}}=0, \\
a_{4,0} \cdot \frac{216}{125}-\frac{2^{7}}{5^{4}} a_{5,1}+4 a \frac{b_{3,8}}{b_{3,3}}-9 a \frac{b_{4,9}}{b_{4,4}}=0, \\
a_{4,0} \cdot \frac{288}{125}-\frac{2^{5}}{125} a_{5,1}+5 a \frac{b_{4,9}}{b_{4,4}}-10 a \frac{b_{5,10}}{b_{5,5}}=0 .
\end{array}
$$

Eliminating $b_{2,7} / b_{2,2}, b_{3,8} / b_{3,3}, b_{4,9} / b_{4,4}, b_{5,10} / b_{5,5}$ from (55), (87) and (93) we obtain

$$
3^{8} \cdot 8189 a_{4,0}-508 a_{5,1}=0, \quad a_{5,1}-14 a / 5-28179 a_{4,0} / 2=0,
$$

whence

$$
a_{4,0}=D_{1} a, \quad a_{5,1}=D_{2} a,
$$

where $D_{1}$ and $D_{2}$ are constants.

In summary, we have simplified (63) and (67) as

and

$$
\begin{aligned}
& x_{2}=8 x_{1}^{2} / 5+b_{2,7} x_{1}^{7}+b_{2,8} x_{1}^{8}+(9), \\
& x_{3}=48 x_{1}^{3} / 25+b_{3,8} x_{1}^{8}+b_{3,9} x_{1}^{9}+(10), \\
& x_{4}=72 x_{1}^{4} / 125+b_{4,9} x_{1}^{9}+b_{4,10} x_{1}^{10}+(11), \\
& x_{5}=2^{5} x_{1}^{5} / 5^{4}+b_{6,10} x_{1}^{10}+b_{6,11} x_{1}^{11}+(12)
\end{aligned}
$$




$$
\begin{aligned}
\frac{d z}{d u}=a z_{1}, & \frac{d z_{1}}{d u}=a\left(I_{1} z+I_{2} z_{1}+\frac{16}{5} z_{2}\right), \\
\frac{d z_{2}}{d u}=a\left(\frac{1}{2} I_{1} z_{1}+2 I_{2} z_{2}+\frac{18}{5} z_{3}\right), & \frac{d z_{3}}{d u}=a\left(\frac{1}{2} I_{1} z_{2}+3 I_{2} z_{3}+\frac{6}{5} z_{4}\right), \\
\frac{d z_{4}}{d u} & =a\left(D_{1} z+\frac{4}{3} I_{1} z_{3}+4 I_{2} z_{4}+\frac{4}{9} z_{5}\right), \\
\frac{d z_{5}}{d u} & =a\left(I_{3} z+D_{2} z_{1}+\frac{9}{4} I_{1} z_{4}+5 I_{2} z_{5}\right) .
\end{aligned}
$$

From the condition of immovability we obtain that

$$
\begin{aligned}
2{ }^{8} I_{3} / 5^{5}-5 b_{2,7}-8 b_{2,8} & =0 \\
6 \cdot 2{ }^{9} I_{3} / 5^{6}-5 I_{2} b_{3,8}+18 b_{2,8} / 5-9 b_{3,9} & =0 \\
3^{3} \cdot 2^{8} I_{3} / 5^{7}-5 I_{2} b_{4,9}+6 b_{3,9} / 5-10 b_{4,10} & =0 \\
4 b_{4,10} / 9-5 I_{2} b_{5,10}-11 b_{5,11} & =0 .
\end{aligned}
$$

Eliminating $b_{i, i+j}(i=2,3,4,5 ; j=5,6)$ from (89) and (96) we obtain

$$
-135 I_{2}\left(1557 a_{4,0}-172 a_{5,1}\right)+I_{3}(36 \cdot 511+55 \cdot 973 / 2)=0 .
$$

Hence follow the projective Frenet-Serret formulae:

$$
\begin{aligned}
\frac{d z}{d \sigma}=z_{1}, & \frac{d z_{1}}{d \sigma}=\left(I_{1} z+I_{2} z_{1}+\frac{16}{5} z_{2}\right), \\
\frac{d z_{2}}{d \sigma}=\left(\frac{1}{2} I_{1} z_{1}+2 I_{2} z_{2}+\frac{18}{5} z_{3}\right), & \frac{d z_{3}}{d \sigma}=\left(\frac{1}{2} I_{1} z_{2}+3 I_{2} z_{3}+\frac{6}{5} z_{4}\right), \\
\frac{d z_{4}}{d \sigma} & =\left(D_{1} z+\frac{4}{3} I_{1} z_{3}+4 I_{2} z_{4}+\frac{4}{9} z_{5}\right), \\
\frac{d z_{5}}{d \sigma} & =\left(D_{3} I_{2} z+D_{2} z_{1}+\frac{9}{4} I_{1} z_{4}+5 I_{2} z_{5}\right),
\end{aligned}
$$

where $D_{1}, D_{2}, D_{3}$ are constants.

In this case five points can be taken for the unit point. $I_{1}, I_{2}$ and $d \sigma$ are uniquely determined save for a constant factor.

4.4. The case where every point of $\Gamma$ is an 8-ic point of the curve $C$. The necessary and sufficient condition that the analytic point $P$ of $C$ is an 8-ic point are $\omega_{1}=0,(85)$ and

$$
2\left(B_{0} D_{0} / C_{0}\right) C_{5}-B_{0} D_{5}-B_{5} D_{0}=0 .
$$

Now the covariant vertex $P_{2}$ can be determined only by the projective differential theory of 8 -ic points. In case that the triangle of reference $\left\{P P_{1} P_{2}\right\}$ 
and the unit point $P+P_{1}+P_{2}$ are selected as in $\S 2.2$, the quartic

$$
X_{2}^{4}+4 X_{0}^{2}\left(X_{1}^{2}-X_{0} X_{2}\right)=0
$$

must have at $P$ a contact of order nine with $C$. Consequently

$$
\begin{gathered}
C_{0}^{2}-B_{0} D_{0}=0, \quad D_{0}^{4}+4 B_{0}^{2}\left(2 C_{0} C_{6}-B_{0} D_{6}-B_{6} D_{0}\right)=0, \\
2 C_{0} C_{7}-B_{0} D_{7}-B_{7} D_{0}=0 .
\end{gathered}
$$

In virtue of (7), (89) and

$$
\begin{aligned}
& B_{7}=\prod_{3}^{5} b_{i, i}\left[6000 \frac{b_{3,10}}{b_{3,3}}-2^{4} \cdot 990 \frac{b_{4,11}}{b_{4,4}}+2^{7} \cdot 3^{4} \frac{b_{5,12}}{b_{5,5}}\right], \\
& C_{7}=\prod_{3}^{5} b_{i, i}\left[3240 \frac{b_{3,10}}{b_{3,3}}-7680 \frac{b_{4,11}}{b_{4,4}}+176 \cdot 3^{3} \frac{b_{5,12}}{b_{5,5}}\right], \\
& D_{7}=\prod_{2}^{5} b_{i, i}\left[-480 \frac{b_{2,9}}{b_{2,2}}+1440 \frac{b_{3,10}}{b_{3,3}}-81 \cdot 32 \frac{b_{4,11}}{b_{4,4}}+1440 \frac{b_{5,12}}{b_{5,5}}\right],
\end{aligned}
$$

the equations (99) reduce to

$$
b_{2,2}=8 / 5 \text {, }
$$

$$
8 / 5^{6}+25 b_{3,9} / b_{3,3}-105 b_{4,10} / b_{4,4}+(252 / 5) b_{5,11} / b_{5,5}+60 b_{2,8} / b_{2,2}=0
$$

and

(102) $-155 b_{3,10} / b_{3,3}+80 b_{2,9} / b_{2,2}+244 b_{4,11} / b_{4,4}+(126 / 5) b_{5,12} / b_{5,5}=0$

respectively. (77), (81) and (92) still remain valid, but the second equation of (94) should be replaced by

$$
2 a_{5,1}-28179 a_{4,0}=0,
$$

because of replacing (87) by (97). Hence

$$
a_{5,1}=0, \quad a_{4,0}=0 ;
$$

and a priori,

$$
b_{2,7}=0, \quad b_{3,8}=0, \quad b_{4,9}=0, \quad b_{5,10}=0 .
$$

The equations (96) then reduce to

$$
\begin{aligned}
& 2^{5} I_{3}-5^{5} b_{2,8}=0, \quad 2^{10} I_{3}+6 \cdot 5^{5} b_{2,8}-3 \cdot 5^{6} b_{3,9}=0, \\
& 3^{3} \cdot 2^{7} I_{3}+3 \cdot 5^{6} b_{3,9}-5^{8} b_{4,10}=0, \quad 4 b_{4,10} / 9-11 b_{5,11}=0 .
\end{aligned}
$$

Eliminating $b_{2,8}, b_{3,9}, b_{4,10}$ and $b_{5,11}$ from (101) and (104) we find

$$
I_{3}=198 a 5 \cdot 529 \cdot 673 \text {. }
$$


The conditions of immovability imply

$$
\begin{gathered}
2 I_{2} b_{2,8}+3 b_{2,9}=0, \quad 3 I_{2} b_{3,9}+5 b_{3,10}-9 b_{2,9} / 5=0, \\
6 I_{2} b_{4,10}+11 b_{4,11}-6 b_{3,10} / 5=0, \quad 6 I_{2} b_{5,11}+12 b_{5,12}-4 b_{4,11} / 9=0,
\end{gathered}
$$

which cannot be consistent with (102) unless

$$
I_{2}=0 \text {. }
$$

In summary, we obtain the canonical expansions of $\Gamma$ :

$\left(63^{\prime \prime}\right)$

$$
\begin{aligned}
& x_{2}=8 x_{1}^{2} / 5+b_{2,8} x_{1}^{8}+b_{2,10} x_{1}^{10}+(11), \\
& x_{3}=48 x_{1}^{3} / 25+b_{3,9} x_{1}^{9}+b_{3,11} x_{1}^{11}+(12), \\
& x_{4}=72 x_{1}^{4} / 125+b_{4,10} x_{1}^{10}+b_{4,12} x_{1}^{12}+(13), \\
& x_{5}=2^{5} x_{1}^{5} / 5^{4}+b_{5,11} x_{1}^{11}+b_{5,13} x_{1}^{13}+(14),
\end{aligned}
$$

where $b_{2,8}, b_{3,9}, b_{4,10}$ and $b_{5,11}$ are constants given by (104) and (105), and the projective Frenet-Serret formulae:

(E)

$$
\begin{aligned}
& \frac{d z}{d \sigma}=z_{1}, \quad \frac{d z_{1}}{d \sigma}=I_{1} z+\frac{16}{5} z_{2}, \quad \frac{d z_{2}}{d \sigma}=\frac{I_{1}}{2} z_{1}+\frac{18}{5} z_{3}, \\
& \frac{d z_{3}}{d \sigma}=\frac{1}{2} I_{1} z_{2}+\frac{6}{5} z_{4}, \quad \frac{d z_{4}}{d \sigma}=\frac{4}{3} I_{1} z_{3}+\frac{4}{9} z_{5} \text {, } \\
& \frac{d z_{5}}{d \sigma}=\frac{198}{5 \cdot 529 \cdot 673} z+\frac{9}{4} I_{1} z_{5} .
\end{aligned}
$$

4.5. The case where every point of $\Gamma$ is a 9-ic point of the curve $C$. We are now in a position to deal with the remaining case. In the same manner as before $P P_{1}$ is taken as the tangent to $\Gamma$ at $P$, and $P_{1} P_{2}$ as the tangent to the osculating conic at one of its points $P_{2}$, and $P+P_{1}+P_{2}$ on the osculating conic.

When every point $P$ of $\Gamma$ is a 9 -ic point of $C$, the equations $\omega_{1}=0,(85)$, (97) and

$$
2 C_{0} C_{6}-B_{0} D_{6}-B_{6} D_{0}=0
$$

must be consistent. After multiplying the coordinates of the points $(z),\left(z_{1}\right)$, $\cdots,\left(z_{5}\right)$ with suitable factor $\rho(u)$, the equations (75) remain valid. Because (77), (81), (92) and (103) are satisfied here, (63) and (67) become

$$
\begin{array}{ll}
x_{2}=\frac{8}{5} x_{1}^{2}+\sum_{l=8}^{\infty} b_{2,2+l} x_{1}^{2+l}, & x_{3}=\frac{48}{25} x_{1}^{3}+\sum_{l=8}^{\infty} b_{3,3+l} x_{1}^{3+l}, \\
x_{4}=\frac{72}{125} x_{1}^{4}+\sum_{l=8}^{\infty} b_{4,4+l} x_{1}^{4+l}, & x_{5}=\frac{2^{5}}{5^{4}} x_{1}^{5}+\sum_{l=8}^{\infty} b_{5,5+l} x_{1}^{5+l},
\end{array}
$$

and 


$$
\begin{array}{ll}
\frac{d z}{d u}=a z_{1}, & \frac{d z_{1}}{d u}=a\left(I_{1} z+I_{2} z_{1}+\frac{16}{5} z_{2}\right), \\
\frac{d z_{2}}{d u}=a\left(\frac{I_{1}}{2} z+2 I_{2} z_{2}+\frac{18}{5} z_{3}\right), & \frac{d z_{3}}{d u}=a\left(\frac{I_{1}}{2} z_{1}+3 I_{2} z_{3}+\frac{6}{5} z_{4}\right), \\
\frac{d z_{4}}{d u}=a\left(\frac{4}{3} I_{1} z_{3}+4 I_{2} z_{4}+\frac{4}{9} z_{5}\right), & \frac{d z_{5}}{d u}=a\left(\frac{9 I_{1}}{4} z_{4}+5 I_{2} z_{5}\right) .
\end{array}
$$

We have to treat particularly the condition of immovability

$$
\frac{d}{d u}\left(z+\sum_{j=1}^{5} x_{j} z_{j}\right)=\lambda(u)\left(z+\sum_{j=1}^{5} x_{j} z_{j}\right)
$$

Substituting (110) in it,

$$
\begin{aligned}
\lambda & =a I_{1} x_{1}, \\
\lambda x_{2} & =\frac{16}{5} a x_{1}+\frac{d x_{2}}{d u}+2 a I_{2} x_{2}+\frac{a I_{1}}{2} x_{3}, \\
\lambda x_{3} & =\frac{18}{5} a x_{2}+\frac{d x_{3}}{d u}+3 a I_{2} x_{3}+\frac{a I_{1}}{3} x_{4}, \\
\lambda x_{4} & =\frac{6}{5} a x_{3}+\frac{d x_{4}}{d u}+4 a I_{2} x_{4}+\frac{a I_{1}}{4} x_{5}, \quad \lambda x_{5}=\frac{a}{9} a x_{4}+\frac{d x_{5}}{d u}+5 a I_{2} x_{5},
\end{aligned}
$$

and eliminating $\lambda$ and $x_{2}$ from the first two equations of (111), we obtain

$$
\frac{d x_{1}}{d u}=-a-a I_{2} x_{1}+\frac{9}{5} a I_{1} x_{1}^{2}+\frac{a I_{1}}{2} \sum_{l=8}^{\infty} b_{2,2+l} x_{1}^{2+l} \text {. }
$$

Therefore the third of (111) is found to be equivalent to

$$
\begin{aligned}
& I_{1} a x_{1}\left(\frac{8}{5} x_{1}^{2}+\right.\left.\sum_{l=8}^{\infty} b_{2,2+l} x_{1}^{2+l}\right)=\frac{16}{5} a x_{1}+2 a I_{2}\left(\frac{8}{5} x_{1}^{2}+\sum_{l=8}^{\infty} b_{2,2+l} x_{1}^{2+l}\right) \\
&+ {\left[\frac{16}{5} x_{1}+\sum_{l=8}^{\infty}(2+l) b_{2,2+l} x_{1}^{1+l}\right] } \\
& \cdot\left[-a-a I_{2} x_{1}+\frac{9 a}{5} I_{1} x_{1}^{2}+\frac{a I_{1}}{2} \sum_{l=8}^{\infty} b_{2,2+l} x_{1}^{2+l}\right] \\
&+\frac{a I_{1}}{2}\left[\frac{48}{25} x_{1}^{3}+\sum_{8}^{\infty} b_{3,3+l} x_{1}^{3+l}\right]
\end{aligned}
$$

on account of $\lambda=a x_{1} I_{1},(112)$ and the first two equations of (109). Comparing 
in (113) the coefficients of equal powers in $x_{1}$, we see that $I_{1}$ and $b_{2,2+l}$ $(l=8,9, \ldots)$ vanish. From the last three equations of (111), we find similarly that

$$
b_{3,3+l}=0, \quad b_{4,4+l}=0, \quad b_{5,5+l}=0 \quad(l=8,9, \cdots) .
$$

Finally, the expansions of $\Gamma$ become

$$
x_{2}=8 x_{1}^{2} / 5, \quad x_{3}=48 x_{1}^{3} / 25, \quad x_{4}=72 x_{1}^{4} / 125, \quad x_{5}=2^{5} x_{1}^{5} / 5^{2} .
$$

We may easily find the geometrical construction of them by extending the method used for a space curve in ordinary space.

4.6. The geometrical interp retation of the invariants and the arc-element. The unit point geometrically defined in the foregoing sections is available in the interpretation of the invariants and the arc-element. In fact, projection from $P_{2}$ of the consecutive point of $P$ on $\Gamma$ to the line $P P_{1}$ produces $P+d \sigma P_{1}$ save for infinitesimal of higher order. Evidently

$$
\left(P, P_{1} ; P+d \sigma P_{1}, P+P_{1}\right)=d \sigma .
$$

This furnishes a geometrical interpretation of the projective arc-element.

As to the interpretation of the invariants we utilize the last equation of the Frenet-Serret formulae. For example, from the equation

$$
\frac{d z_{5}}{d \sigma}=I_{4} z+I_{3} z_{1}+\frac{5^{3} \cdot 2699}{2^{5}} I_{2} z_{2}+\frac{291}{40} z_{3}+\frac{9}{4} I_{1} z_{4}-\frac{5^{4} \cdot 3^{3}}{2} I_{2} z_{5},
$$

it can be shown that the line $P P_{3}$ intersects the hyperplane containing $P_{1}, P_{2}$, $P_{4}$ and the tangent at $P_{5}$ to the curve $\left(P_{5}\right)$ at the point $I_{4} P+291 P_{1} / 40$ and the double ratio $\left(P, P_{3} ; P+P_{3}, I_{4} P+291 P_{3} / 40\right)$ equals, except for a constant factor, the invariant $I_{4}$. Since other invariants may be similarly interpreted we omit their interpretations here.

\section{BIBLIOGRAPHY}

1. Bompiani, Per lo studio proiettivo-differenziale della singolaritd, Boll. dell'Unione Mat. Italiana vol. 5 (1926) pp. 118-120.

2. Chang, $A$ generalization of sextactic points of a plane curve, not yet published.

3. - On the point of inflexion of a plane curve, Duke Math. J. vol. 9 (1942) pp. 823-832.

4. - On the point of inflexion of a space curve, To be published in Revista de Tucumàn, Argentina.

5. - On the singularity $S_{1}^{4}$ of a plane curve, not yet published.

6. - - On the singularity $S_{1}^{m}$ of a plane curve, Duke Math. J. vol. 9 (1942) pp. 833-845.

7. - Researches on the projective differential theory of singular points, I, II, to be published in Amer. J. Math.

8. Lane, The neighborhood of a sextactic point on a plane curve, Duke Math. J. vol. 1 (1935) pp. 287-292.

9. Levi-Civita and Fubini, Sulle curve analogue al circolo osculatore quando si passa da tre a quattro punti infinitamente vicini, Annali di Mat. (4) vol. 7 (1929) pp. 193-211. 
10. Sannia, Nuova trattazione della geometria proiettiva differenziale delle curve sghembe, Memoir I, Annali di Matematica (4) vol. 1 (1924) pp. 1-18; Memoir II, ibid. vol. 3 (1926) pp. 1-25.

11. Su, Contributions to the projective theory of curves in space of five dimensions, to be published in Duke Math. J.; an abstract of this paper will appear in Science Record, Academia Sinica.

12. - Note on the projective differential geometry of space curves, Journal of the Chinese Mathematical Society vol. 1 (1937) pp. 98-137.

13. Vychyclo, Interpretazione geometrica della curvatura proiettiva della curve plane, Bollettino dell'Unione Matematica I taliana vol. 17 (1938) pp. 74-77.

14. - Sur une interprétation géométrique de la courbure projective des courbes planes, Bull. Soc. Math. France vol. 64 (1936) pp. 87-98.

15. Wilczynski, Projective differential geometry of curves and ruled surfaces, Teubner, 1906.

National University of Chekiang, Meitan, Kweichow, China. 